my journal manuscript No.

(will be inserted by the editor)

\title{
GRB Observational Properties
}

\author{
Bing Zhang · Hou-Jun Lü • En-Wei Liang
}

Received: date / Accepted: date

\begin{abstract}
We summarize basic observational properties of gamma-ray bursts (GRBs), including prompt emission properties, afterglow properties, and classification schemes. We also briefly comment on the current physical understanding of these properties.
\end{abstract}

Keywords Gamma-ray bursts

\section{Introduction}

Gamma-ray bursts (GRBs) are energetic bursts of $\gamma$-rays from deep space. They are the most luminous objects in the universe, signifying births of stellar-mass black holes or millisecond magnetars in most violent explosions, such as core collapse of rapidly spinning massive stars or mergers of two compact stars (NSNS or NS-BH). Many in-depth reviews have been written on the subject: e.g. (Fishman and Meegan 1995; Piran 1999; van Paradiis et al. 2000; Mészáros 2002; Zhang and Mészáros 2004; Piran 2004; Mészáros|2006; Zhang| 2007; Gehrels et al. 2009; Kumar and Zhang 2015). This chapter summarizes the basic observational properties of GRBs, including prompt emission (22), afterglow (\$3), and classification schemes (4). Besides outlining the observational facts, a brief discussion on the current physical understanding of the data is also presented.

Bing Zhang

Department of Physics and Astronomy, University of Nevada Las Vegas, NV 89154, USA

E-mail: zhang@physics.unlv.edu

Hou-Jun Lü

GXU-NAOC Center for Astrophysics and Space Sciences, Department of Physics, Guangxi University, Nanning 530004, China

Guangxi Key Laboratory for Relativistic Astrophysics, Nanning, Guangxi 530004, China

E-mail: lhj@gxu.edu.cn

En-Wei Liang

GXU-NAOC Center for Astrophysics and Space Sciences, Department of Physics, Guangxi University, Nanning 530004, China

Guangxi Key Laboratory for Relativistic Astrophysics, Nanning, Guangxi 530004, China

E-mail: lew@gxu.edu.cn 


\section{Prompt emission}

GRB prompt emission is usually defined as the soft $\gamma$-ray / hard X-ray emission detected by the GRB triggering detectors (such as Swift/BAT and Fermi/GBM). Some GRBs are detected in higher (e.g. GeV) or lower (e.g. optical) energies during the prompt emission phase.

\subsection{Temporal properties}

\subsubsection{Duration distribution}

GRB duration is usually quantified as $T_{90}$, the duration during which $5 \%$ to $95 \%$ of the total fluence is detected by the triggering detector. The definition of $T_{90}$ depends on the energy band and sensitivity of the detector. A same burst may have a longer $T_{90}$ if the detector is more sensitive or has a softer energy band.

In the CGRO/BATSE band (25-350 keV), GRBs showed a bimodal distribution with a rough separation at $2 \mathrm{~s}$ (Kouveliotou et al. 1993). This was the foundation of classifying GRBs into long $\left(T_{90}>2 \mathrm{~s}\right)$ and short $\left(T_{90}<2 \mathrm{~s}\right)$ categories (Fig.1a). The bimodal distribution was confirmed by other later detectors (e.g., Sakamoto et al. 2011; Paciesas et al. 2012; Zhang et al. 2012; Lien et al. 2016), even though the relative fractions of the two types vary for different detectors and also for different energy bands in the same detector (Qin et al. 2013). The bimodal classification is more evident in the two-dimensional $T_{90}-\mathrm{HR}$ domain, where HR is the hardness ratio of a burst. Long GRBs are typically softer than short GRBs (Fishman and Meegan 1995, see Fig.1b). Broad-band observations now show that the two duration classes roughly correspond to two types of progenitor systems: long GRBs are related to deaths of massive stars, and short GRBs are related to mergers of compact objects (see 4 for more discussion).

\subsubsection{Lightcurves}

GRB lightcurves are erratic, usually display overlapping emission episodes with a wide range of variability time scales (Fishman and Meegan 1995). Some bursts have one or several clearly separated "pulses", which typically show a fast-rising and exponential-decay (FRED) shape. The duration of these pulses is typically seconds. Some GRBs have a "precursor" followed by a quiescent gap before the main burst comes out. The emission properties of the precursor is not too different from the main burst (e.g., Lazzati 2005; Burlon et al. 2008; Hu et al. 2014). A power density spectrum (PDS) analysis does not reveal quasi-periodic oscillations, but rather show a featureless power law Beloborodov et al. 2000; Guidorzi et al. 2012). However, there is evidence of the superposition of a "fast" variability component on top of a "slow" variability component (Gao et al. 2012). 


\subsection{Spectral properties}

\subsubsection{Spectral models and multiple components}

When enough photons are detected (large enough of GRB fluence), the GRB spectra are usually fit by a phenomenological model called the "Band" function (Band et al. 1993). It is essentially a broken power law model with a smooth (exponential) transition between the two segments. The typical low-energy and high-energy photon indices are $\alpha \sim-1 \pm 1$ and $\beta \sim-2_{-2}^{+1}$ for the GRBs observed with BATSE in the 20-2000 keV band (Preece et al. 2000), and confirmed for GRBs detected by Fermi/GBM and Integral (Zhang et al. 2011; Nava et al. 2011; Gruber et al. 2014). The peak energy of a GRB (when $\beta<-2$ is satisfied) is defined by $E_{p}=(2+\alpha) E_{0}$, where $E_{0}$ is the break energy in the photon spectrum of the Band function. The distribution of $E_{p}$ is about several hundred $\mathrm{keV}$ for bright GRBs, but can vary from several keV for GRB 060218 (Campana et al. 2006) to $15 \mathrm{MeV}$ for GRB 110721A (Axelsson et al. 2012). The spectra of some GRBs may be fit with a cut-off power law, and some others with a single powerlaw function if the GRB is not bright enough or it is observed with a narrow band instrument such as Swift BAT, even though the true spectral shape may be Band-like (Zhang et al. 2007a).

In some GRBs, the superposition of multiple spectral components was observed. A thermal like spectral component claimed in the pre-Fermi era Ryde 2005) was confirmed in the Fermi era. First, GRB 090902B was found to have a narrow Band component superposed on a power law component Abdo et al. 2009a). The narrow Band component could be also fit as a multi-color blackbody (Ryde et al. 2010). When the time bin is small enough, the data could be even fit with a blackbody plus power law model (Zhang et al. 2011). A similar case may be made to the short GRB 090510 (Ackermann et al. 2010). Later, a weak thermal component was suggested to exist in the low-energy shoulder of the Band component of some GRBs (e.g., Guiriec et al. 2011, 2013, 2015; Axelsson et al. 2012). Some GRBs, on the other hand, show featureless Band-like spectra without the need of a thermal component (e.g.,GRB 130606B, Zhang et al. 2016). Another spectral component is the power law component as seen in some GRBs, e.g. GRB 090902B and GRB 090510. This component extends several orders of magnitude from $\mathrm{keV}$ range sometimes all the way to the $\mathrm{GeV}$ range. It usually has a positive slope in $\nu F_{\nu}$ spectral representation, which means that there should be a high-energy $E_{p}$ in the GeV range. Indeed, GRB 090926 was found to have such a component with a high-energy cutoff (Ackermann et al. 2011). Some example GRB spectra are presented in Figure 2. Overall, GRB prompt emission spectra may have three distinct components (Zhang et al. 2011, Fig.2d): I. a Band component; II. a thermal component; and III. a power law component. Different spectral components may dominate in different GRBs. Indeed GRB spectral with different combinations of these three components have been observed. Guiriec et al. (2015) showed that all three components may exist in at least some bright GRBs.

\subsubsection{Spectral evolution}

Prompt emission spectra rapidly evolve with time. This can be seen from two different aspects. First, if one displays lightcurves in different energy bands, one 
would see significant "spectral lags", i.e. a pulse observed in a softer band is usually broader than and lagged behind the same pulse observed in a harder band (Norris et al. 2000; Liang et al. 2006a). Second, if one displays time-dependent spectra, one would see that $E_{p}$ rapidly evolves with time. There are two types of evolution patterns (Norris et al. 1986; Golenetskii et al. 1983; Lu et al. 2010, 2012): hard-to-soft evolution and intensity tracking.

\subsection{Prompt emission in other wavelengths}

During the prompt emission phase, some GRBs are also detected in other energy bands. In the high-energy regime, $\mathrm{GeV}$ emission was detected in the prompt emission phase of several GRBs. In general, GeV emission is delayed with respect to the sub-MeV emission (e.g., Abdo et al. 2009a, b). Sometimes the GeV emission peak seems to align with some sub-MeV emission peaks, but in most cases, the $\mathrm{GeV}$ emission lasts longer than the MeV component (Ghisellini et al. 2010; Zhang et al. 2011), suggesting the emergence of a new emission component.

Prompt optical emission was detected in a few cases. Some GRBs show a tracking behavior between the optical and sub-MeV emission (e.g., the famous "nakedeye" GRB 080319B Racusin et al. 2008), while some others show a distinct peak near the end of prompt emission which is offset from the sub-MeV peaks (e.g., in GRB 990123 Akerlof et al. 1999). The combination of these two components was also seen in some GRBs (e.g. in GRB 050820A Vestrand et al. 2006).

On the other hand, extensive searches of high-energy neutrinos associated with GRBs during prompt emission phase have been carried out, but no detection has been made so far. An increasingly stringent upper limit of the prompt neutrino flux has been placed (Abbasi et al. 2012; Aartsen et al. 2016).

\subsection{Physical understanding}

No general consensus is reached in the community regarding how prompt emission properties are interpreted. This is because there are some uncertainties in the physical models of GRB prompt emission (Zhang 2011, for a review): jet composition (matter-dominated fireball or Poynting-flux-dominated outflow), energy dissipation mechanism and particle acceleration mechanism (internal shocks or magnetic reconnection), and radiation mechanism (synchrotron radiation or Comptonization of quasi-thermal photons). As a result, many models have been proposed to interpret GRB prompt emission. According to the location of emission site, these models may be grouped into three types: 1 . the dissipative photosphere models (e.g., Rees and Mészáros 2005; Pe'er et al. 2006; Giannios and Spruit 2007; Beloborodov 2010; Lazzati and Begelman 2010; Toma et al. 2011; Murase et al. 2012, ; which interpret prompt emission as Comptonized quasi-thermal photons from the photosphere); 2. the internal shock models (e.g., Rees and Meszaros 1994; Daigne and Mochkovitch 1998; Daigne et al. 2011, ; which interpret prompt emission as synchrotron radiation of electrons accelerated in the internal shocks); and 3. the forced magnetic dissipation models with large emission radii (e.g.,the internal collision-induced magnetic reconnection and turbulence, ICMART, model of Zhang and Yan 2011, which interpret prompt emission as synchrotron radiation 
of electrons accelerated in the magnetic dissipation region). These models have different magnetization parameter $\sigma$ in the emission region. The first two models have $\sigma \ll 1$, while the third model has $\sigma \geq 1$ in the emission region 1

The erratic lightcurves of GRB prompt emission are interpreted differently in the three models. In the photosphere models, all the variabilities are directly produced by the generalized central engine (including the central black hole and the stellar envelope through which the jet propagates). The rapid variabilities may directly reflect the intrinsic fluctuation of the jet power from the central black hole, while the slow component may be caused by the modulation of the stellar envelope as the jet emerges from the central engine (Morsony et al. 2010). In the internal shock models, the observed emission is from many internal shocks, some at small radii (the fast component), and some at large radii (the slow component) (Hascoët et al. 2012). In the ICMART model, the slow component reflects the history of central engine activity. Each broad pulse is from one single emission unit (one ICMART event), which gives a seconds-long duration pulse as the emission region moves forward. The rapid variabilities may be caused by the mini-jets produced in the local reconnection regions within the jet (Zhang and Zhang 2014).

It is agreed among the modelers that the thermal component observed in the spectra of some GRBs is emission from the outflow photosphere. The origin of the most common Band component is, however, subject to debate. One view is that it is also emission from the photosphere. The other view is that it is synchrotron emission from an optically thin region (internal shocks or ICMART regions). The simplest version of both models have trouble to interpret the typical low-energy photon index $\alpha \sim-1$ : The photosphere model typically predicts $\alpha \sim+1.4$ (Beloborodov 2010; Deng and Zhang 2014), whereas the synchrotron model typically predicts $\alpha \sim-1.5$ due to fast cooling of the electrons. In order achieve $\alpha \sim-1$, the photosphere model needs to introduce a special type of structured jet model (Lundman et al. 2013). Within the synchrotron model, $\alpha \sim-1$ (or an even harder spectrum) could be achieved if one considers the global decay of the magnetic field strength in the emission region, as is naturally expected in an expanding shell (Uhm and Zhang 2014a). The superposition of a thermal component on top of a Band component poses a challenge to the photosphere mode 2 . Within the internal shock and ICMART models, the Band component can be interpreted as the synchrotron radiation from an optically thin emission region (internal shocks or ICMART region), while the thermal component is the emission from the photosphere. Different bursts have different relative strength between the thermal and non-thermal components. This could be understood within the framework of a hybrid central engine (Gao and Zhang 2015) characterized by different dimensionless entropy $\eta$ (for the fireball component) and magnetization parameter $\sigma_{0}$ (the Poynting flux component). The power law component extending from low energy to very high energy is not easy to explain. One possibility is that it actually includes two emission components, i.e. a low energy synchrotron com-

1 The so-called electromagnetic model (Lyutikov and Blandford 2003) conjectures $\sigma \gg 1$ in the emission region which is close to the deceleration radius. It is hard to maintain such a huge $\sigma$ at the deceleration radius. The detections of the thermal components also disfavor this possibility.

2 Vurm et al. (2011) interprets the Band component as the photosphere emission while the "thermal" bump as the synchrotron radiation. 
ponent and a high-energy inverse Compton component. With certain parameters they may form an effective power law by coincidence (Pe'Er et al. 2012).

Spectral evolution carries important clues regarding the origin of prompt emission. Spectral lags and $E_{p}$ evolution patterns all concern the broad pulses in GRB lightcurves rather than the rapid variabilities. This suggests that each broad pulse reflects emission from one single emitting shell which travels in space. Uhm and Zhang 2016a) showed that the properties of spectral lags can be reproduced within this picture given that the emission radius is large, the magnetic field strength in the emission region is decaying (as expected), and the emission region is undergoing bulk acceleration. The last requirement is consistent with dissipation of magnetic fields in a Poynting-flux-dominated outflow. The same theoretical framework can naturally explain $E_{p}$ hard-to-soft evolution (Uhm and Zhang 2014a) and intensity tracking (Uhm and Zhang 2016b, in preparation). It is very difficult to interpret broad-pulse spectral lags and $E_{p}$ evolution patterns within the photosphere models and internal shock models. The stringent upper limits of neutrino flux during prompt emission phase is also consistent with the picture that most GRBs have a Poynting-flux-dominated jet composition (Zhang and Kumar 2013; Aartsen et al. 2016).

\section{Afterglow}

GRB afterglow is the broad-band emission detected after the prompt emission phase. It is characterized by a multi-segment broken power-law spectrum at a given instant, and a multi-segment broken power law decaying lightcurve in a given observational band. The afterglow was theoretically predicted (Mészáros and Rees 1997) before the first discoveries (Costa et al. 1997; van Paradijs et al. 1997; Frail et al. 1997). After two decades of observations, broad-band afterglows are well studied phenomenologically and physically.

\subsection{X-ray afterglow}

GRB X-ray afterglow was first discovered with BeppoSAX for GRB 970228 (Costa et al. 1997). A systematic study of X-ray afterglows started in the Swift era, when Swift/XRT regularly observe the GRB source starting from tens of seconds after the trigger. A canonical lightcurve can describe the X-ray afterglow lightcurves of most GRBs, which include five components (Zhang et al. 2006; Nousek et al. 2006): a steep decay phase, a shallow decay phase (or plateau), a normal decay phase, a late jet-break-like steepening, and erratic X-ray flares (Fig 3). A small fraction of XRT lightcurves show a monotonic decay with a single power law (Liang et al. 2009). Empirically, O'Brien et al. (2006) and Willingale et al. (2007) showed that the X-ray afterglow data can be fit by the superposition of a prompt emission component and an afterglow component, even though no physical model predicts the specific mathematical forms of the model. Some correlations between X-ray afterglow properties and prompt $\gamma$-ray properties have been found (Grupe et al. 2013).

The observational properties of the five afterglow components may be summarized as follows: 
- Steep decay phase: The temporal decay slope of this segment is usually steeper than -3 , sometimes even as steep as -10 . This segment is usually smoothly connected to the last prompt emission pulse, suggesting that it is the tail emission of prompt emission (Barthelmy et al. 2005). Strong spectral softening is usually observed during the steep decay phase (Zhang et al. 2007; Campana et al. 2006; Mangano et al. 2007; Butler and Kocevski 2007). The origin of the steep decay phase is usually attributed to the high-latitude "curvature effect", with a proper zero time adopted (Kumar and Panaitescu 2000; Zhang et al. 2006; Liang et al. 2006b). The rapid spectral evolution requires a curved spectrum at the end of prompt emission (Zhang et al. 2007, 2009).

- Shallow decay phase (plateau): This segment is usually seen in the early XRT lightcurves following the steep decay phase. Its decay slope ranges from 0 to -0.5 and the typical duration of this segment is $\sim 10^{4}$ seconds with a fluence of $\sim 3 \times 10^{-7} \mathrm{erg} / \mathrm{s}$ in the XRT band (Liang et al. 2007). The typical photon index is $\sim 2.1$ (O'Brien et al. 2006; Liang et al. 2007), and there is no spectral evolution seen across the break from this segment to the normal segment afterwards (Liang et al. 2007). The luminosity at the break time is anti-correlated with the break time in the burst frame (Dainotti et al.|2010; Li et al. 2012). In most cases, the shallow decay phase is followed by a normal decay with flux decreasing with time as $\sim t^{-1}$. These plateaus can be understood within the standard external shock model with continuous energy injection into the blastwave (Zhang et al. 2006; Nousek et al. 2006; Rees and Mészáros 1998; Dai and Lu 1998a, b; Zhang and Yan 2011; Yu and Dai 2007). In a few cases, the shallow decay segment appears as a plateau followed by a very steep decay (with index around -9) in both long and short GRBs (Troia et al. 2007; Liang et al. 2007; Lyons et al. 2010; Rowlinson et al. 2010, 2013). These cases cannot be interpreted within the external shock models, and therefore are termed as "internal plateaus". They demand direct energy dissipation from a long-lasting central engine, likely a millisecond magnetar (Troia et al. 2007; Lvons et al. 2010; Rowlinson et al. 2010, 2013; Lü and Zhang 2014; Lü et al. 2015).

- Normal decay and jet break: The normal decay phase has a typical decay index $\sim-1$, consistent with the prediction of the standard external forward shock model (Mészáros and Rees 1997; Sari et al. 1998). In some GRBs, there is an additional steepening break at later times, after which the decay slope may reach $\sim-2$ (Liang et al. 2008; Racusin et al. 2009). This is consistent with the so-called jet break (Rhoads 1999; Sari et al. 1999). Some bursts were observed with Swift/XRT and/or Chandra weeks and even months after the GRB triggers, with no evidence of detecting a jet break in their X-ray lightcurves (e.g., Grupe et al. 2006).

- X-ray flares: X-ray flares are detected in less than half of Swift GRBs. Their lightcurves show rapid rise and fall Burrows et al. 2005; Falcone et al. 2006; Romano et al. 2006) with strong spectral evolution (Lazzati and Perna 2007; Chincarini et al. 2007; Margutti et al. 2011). The fluence of a flare is usually about $1 \%-10 \%$ of that of prompt emission, but occasionally may be comparable. Temporal and spectral analyses of X-ray flares reveal many properties analogous to prompt emission (Burrows et al. 2005; Chincarini et al. 2010; Margutti et al. 2011). They signify the reactivation of the central engine at later times (Burrows et al. 2005; Fan and Wei 2005; Zhang et al. 2006; Lazzati and Perna 2007). 


\subsection{Optical Afterglow}

In the pre-Swift era, afterglow observations were mostly made in the optical bands after hours of GRB triggers. The late optical light curves usually show a single power law decay with a decay index of $\sim t^{-1}$. Occasionally, a two-segment broken power law from $\sim t^{-1}$ to $\sim t^{-2}$ was observed, with the temporal break defined as a "jet break". In some cases, an early optical flash was detected, which is characterized by a steep decay with $\sim t^{-2}$ (e.g.,GRB 990123 Akerlof et al. 1999) and interpreted as emission from the reverse shock (Mészáros and Rees 1997, 1999; Sari and Piran 1999b, a). Kann et al. (2010, 2011) presented optical afterglow lightcurves in both the observer frame and a common rest-frame at $z=1$ for a large sample of bursts (Fig. 悄). Similar to the X-ray afterglow lightcurves, the optical afterglow lightcurves are also composed of several different emission components ( $\mathrm{Li}$ et al. 2012, Figure 4b): Ia. prompt optical flares; Ib. an early optical flare from the reverse shock; II. early shallow decay segment; III. the standard afterglow component (sometimes led by an afterglow onset hump due to deceleration); IV. the post jet break phase; V. optical flares; VI. rebrightening humps; VII. late supernova (SN) bumps. The components II-V can find their counterparts in the canonical X-ray lightcurve.

One interesting feature in some optical lightcurves is an early hump that marks the deceleration of the ejecta (e.g., Rees and Meszaros 1992; Meszaros and Rees 1993; Sari and Piran 1999a, for the thin shell case, and Kobayashi et al. 1999; Kobayashi and Zhang 2007, for the thick shell case). The peak time in the lightcurve can be used to infer the initial Lorentz factor of the ejecta during the afterglow phase (e.g. Molinari et al. 2007; Xue et al. 2009; Melandri et al. 2010; Liang et al. 2010). A statistical analysis (Liang et al. 2013) suggests that the typical rising and decaying slopes of the onset hump are $\sim 1.5$ and $\sim-1.15$, respectively. The peak luminosity is anti-correlated with the peak time, $L_{p} \propto t_{p}^{-1.81 \pm 0.32}$. Both $L_{p}$ and the isotropic energy release of the onset bumps are correlated with $E_{\gamma \text {,iso. }}$. Liang et al. (2010) discovered a correlation between $\Gamma_{0}$ and $E_{\gamma, \text { iso }}$ for a constant density medium. A similar relation between $\Gamma_{0}$ and $\gamma$-ray isotropic luminosity was also claimed (Ghirlanda et al. 2011; Lü et al. 2012).

One important subject is the "chromaticity" of the afterglows. Within the standard external forward shock models, optical and X-ray emission comes from the same synchrotron emission component. As a result, the hydrodynamical or geometrical temporal breaks (e.g. the energy injection break and jet break) in the lightcurves should be achromatic, i.e. a break should occur simultaneously in the optical and X-ray bands. However, multi-wavelength observations suggested that some GRBs showed chromatic behaviors in the two bands (e.g., Panaitescu et al. 2006; Fan and Piran 2006; Liang et al. 2007, 2008, Fig 5). A systematic study (Wang et al. 2015) suggested that the majority of afterglows are consistent with being roughly achromatic, suggesting that the standard external shock models can account for most of the afterglow observations.

\subsection{Radio afterglow}

Compared with X-ray and optical afterglows, radio afterglow emission is more difficult to be detected with the available telescopes. About $30 \%$ of GRBs were detected 
in radio, and this rate did not change before or after Swift was launched. Typically radio afterglow light curves at $8.5 \mathrm{GHz}$ show initial rising and a peak time around 3-6 weeks in the rest frame (Fig,6, Chandra and Frail 2012). This is consistent with the standard external forward shock model, with the peak corresponding to the crossing of the typical synchrotron frequency $\nu_{m}$ or the self-absorption frequency $\nu_{a}$ in the radio band. There is a clear relationship between the detectability of the radio afterglow and the fluence of a GRB (Chandra and Frail 2012)(Chandra \& Frail 2012). Some well-monitored bright GRBs show an early radio flare (e.g. GRB 990123, Kulkarni et al. 1999 and GRB 130427A, Anderson et al. 2014), which is usually attributed to the emission from the reverse shock (Sari and Piran 1999b; Kobayashi and Zhang 2003a).

\subsection{High energy afterglow}

Conventionally high-energy emission refer to photons above $100 \mathrm{MeV}$. The first detection of high-energy afterglow was in the Compton Gamma-Ray Observatory (CGRO) era, when GRB 940217 was detected in GeV energies hours after the burst was over (Hurley et al. 1994). Fermi/LAT detected a small fraction of GRBs in the GeV range. Most of these $\mathrm{GeV}$-detected GRBs have their high-energy emission lasting longer than the prompt emission itself, suggesting an afterglow origin (e.g., Abdo et al. 2009a, b; Ackermann et al. 2010, 2011). Systematic studies suggest that the lightcurves typically show a power law decay, with the possibility of an early steep-to-shallow break transition (Ghisellini et al. 2010; Zhang et al. 2011; Ackermann et al. 2013).

\subsection{Physical understanding}

The standard theoretical framework to understand GRB afterglows is the external shock model. As the relativistic ejecta (also termed as fireball) is decelerated by the circumburst medium, either a constant density ISM or a stratified stellar wind, a pair of shocks are developed: one long-lasting forward shock propagating into the medium and a short-lived reverse shock propagating into the ejecta (Meszaros and Rees 1993; Sari and Piran 1995). Electrons are accelerated from both shocks, which radiate broad-band synchrotron radiation with characteristic frequencies evolving with time. The forward shock gives rise to the broad-band long-lasting afterglow (Mészáros and Rees 1997; Sari et al. 1998; Dai and Lu 1998c; Chevalier and Li 2000; Huang et al. 2000) and a short-lived reverse shock may give additional significant optical and radio emission early on (Mészáros and Rees 1997, 1999; Sari and Piran 1999b; a; Kobavashi 2000; Zhang et al. 2003; Kobavashi and Zhang 2003a, b; Wu et al.2003; Fan et al. 2004; Zhang and Kobayashi 2005; Resmi and Zhang 2016). For a comprehensive review of all the external shock models see Gao et al. (2013a). If the central engine is long lasting (Dai and Lu 1998a, b; Zhang and Mészáros 2001, 2002) or if the ejecta has a stratified Lorentz factor distribution (Rees and Mészáros 1998; Sari and Mészáros 2000), continuous energy injection into the blastwave (defined as the region between the forward shock and reverse shock) is possible, so that the lightcurve decay slopes are shallower. In these cases, the reverse shock is long lived. If the reverse shock is 
more magnetized, these long-lasting reverse shocks may be the dominant emitter of afterglow emission (Uhm and Beloborodov 2007; Genet et al. 2007; Uhm et al. 2012).

Over the years, GRB afterglow data have been extensively modeled using the external shock model. In the pre-Swift era, afterglow observations were carried out hours after the bursts, and the data are in general well-interpreted by the models (e.g., Panaitescu and Kumar 2001, 2002; Yost et al. 2003). Swift unveiled the early afterglow phase and discovered some unpredicted features (e.g. the steep decay phase, shallow decay phase, and X-ray flares). The theoretical framework in the post-Swift era invokes emission from both the external shock and internal energy dissipation regions (internal shocks or magnetic dissipation regions) due to the late central engine activities. Shortly after Swift started to detect early afterglow regularly, Zhang et al. (2006) suggested that the steep decay phase and the X-ray flares are of an internal origin, and that the shallow decay, normal decay and late steepening phases are from the external shock with the shallow decay phase being due to continuous energy injection into the blastwave. Such a simple paradigm remains valid to interpret most of GRB afterglows after a decade of observations.

In the optical band, the contamination of internal emission from late central engine activities is less, and most lightcurves can be interpreted within the standard forward shock model. Some bursts have early reverse shock component, but some others do not have evidence of reverse shock emission. The diversity may be attributed to different magnetization degree $\sigma$ of the ejecta. A moderately magnetized reverse shock $(\sigma<0.1)$ is favorable to produce a bright optical flash due to the enhanced synchrotron emission in the reverse shock region (Fan et al. 2002; Zhang et al. 2003; Kumar and Panaitescu 2003), but not necessarily a bright radio flash due to synchrotron self-absorption in the forward shock region (Resmi and Zhang 2016). An ejecta with $\sigma \ll 1$ (weak synchrotron emission) or $\sigma \geq 1$ (weak or no reverse shock) is not expected to produce bright reverse shock emission in optical (Zhang and Kobavashi 2005). The early optical afterglow data of a large sample of GRBs are consistent with a simple picture that GRB ejecta have a distribution of the magnetization parameter $\sigma$ among different bursts (Gao et al. 2015).

The chromatic nature of afterglow observed in some GRBs cast doubts on interpreting all the afterglow data using the standard forward shock model. At least two different emission components are needed to interpret the data. One possibility is that long-lasting central engine activities are responsible to the entire observed X-ray afterglow emission (e.g., Ghisellini et al. 2007; Kumar et al. 2008a, b), while the optical emission is from the external forward shock. A second possibility is that a long-lasting reverse shock and the forward shock are contributing emission in different energy bands (Uhm et al. 2012; Uhm and Zhang 2014b). Another possibility is to invoke a two-component jet with each component dominating emission in one observational band (e.g., de Pasquale et al. 2009). A recent systematic study (Wang et al. 2015) suggested that the fraction of GRB afterglows that are consistent with being achromatic is at least $50 \%$ and may be as high as $90 \%$. This suggests that these unconventional models may not be needed to interpret the majority of GRBs.

It is the consensus in the community (Kumar and Barniol Duran 2009, 2010; Zhang et al. 2011; He et al. 2011; Liu and Wang 2011; Maxham et al. 2011) that $\mathrm{GeV}$ afterglow after the prompt emission phase is of an external forward shock 
origin, while $\mathrm{GeV}$ emission during the prompt emission phase may have a contribution from the internal dissipation region. The radiation mechanism is believed to be dominated by synchrotron radiation, although synchrotron self-Compton may have been detected in GRB 130427A (Fan et al. 2013; Liu et al. 2013; Tam et al. 2013; Ackermann et al. 2014).

\section{Classification schemes}

Diverse GRBs with different observational properties have been observed. Classifying GRBs has been a challenging task, and many suggestions have been made. In general, these proposed classification schemes can be divided in two categories: phenomenological classification schemes and physical classification schemes.

\subsection{Phenomenological classification schemes}

The following classification schemes have been proposed in the literature:

- Duration-hardness classification scheme: This is the traditional long/soft vs. short/hard classification scheme (Kouveliotou et al. 1993). The main criterion is $T_{90}$, and hardness ratio is regarded as a supplementary criterion. The measured duration $T_{90}$ depends on the energy band and sensitivity of the detector Qin et al. 2013), which may bring in ambiguity of classification in some bursts. For example, in the Swift era, some short GRBs are detected to be followed by soft extended emission lasting 10's to $\sim 100 \mathrm{~s}$ (Norris and Bonnell 2006). Based on $T_{90}$ distribution information, some authors suggested a third, intermediateduration class (ee.g., Mukherjee et al. 1998; Horváth 1998; Hakkila et al. 2003). An ultra-long population was recently suggested (Gendre et al. 2013; Levan et al. 2014), even though whether it indeed form a distinct category is subject to debate (Virgili et al. 2013; Zhang et al. 2014; Boër et al. 2015; Gao and Mészáros 2015).

- High-luminosity (HL) vs. low-luminosity ( $L L)$ : Long GRBs are sometimes classified into two sub-categories based on their luminosities, with a separation line roughly at $\left(10^{48}-10^{49}\right) \mathrm{erg} \mathrm{s}^{-1}$. The LL-GRBs typically have long durations and smooth lightcurves, and have a much larger local event rate density than the traditional HL-GRBs (Campana et al. 2006; Soderberg et al. 2006; Liang et al. 2007; Virgili et al. 2009; Sun et al. 2015).

- GRBs, X-ray rich GRBs, and X-ray flashes: Based on spectral properties, long GRBs are sometimes further grouped into GRBs, X-ray rich GRBs, and Xray flashes. The separation line is arbitrary since there is no clear bimodal distribution in the hardness distribution. Studies showed that these events form a continuum in their properties (e.g., Sakamoto et al. 2005). Most LL-GRBs are also X-ray flashes.

- Supplementary criteria: Some prompt emission properties can be regarded as supplementary criteria for the duration classification scheme. Besides hardness ratio, spectral lags are often used to define whether a GRB is short (short GRBs typically have negligible spectral lags, e.g. Gehrels et al. 2006). A combination of isotropic energy and rest-frame $E_{p}$ (the so-called $\epsilon=E_{\gamma, \text { iso }} / E_{p, z}^{5 / 3}$ parameter) serves as a good separator between the long and short GRBs (Lü et al. 
2010). Also, the amplitude parameters ( $f$ and $\left.f_{\text {eff }}\right)$ can be very good criteria to distinguish between long and short (Lü et al. 2014).

- Afterglow-based classification schemes: Based on optical afterglow data, GRBs can be classified as optically bright and optically dark GRBs (Jakobsson et al. 2004; Rol et al. 2005). Based on the X-ray afterglow data one may classify GRBs into those with X-ray flares and those without, or those following a canonical lightcurve and those following a single power law decay.

\subsection{Physical classification schemes}

The ultimate goal of studying astrophysical phenomenology is to uncover the physical nature of the sources. Based on observational properties and theoretical modeling of GRBs over the years, GRBs are physically classified into different categories.

- Massive star (Type II) vs. Compact star (Type I) GRBs: The two dominant categories of GRBs are the ones associated with deaths of massive stars and the ones not. The former category roughly correspond to the long duration GRBs, and latter roughly correspond to the short duration GRBs. However, counter examples do exist, e.g. SN-less long duration GRBs (GRB 060614 and GRB 060505) that are likely associated with compact stars (Gehrels et al. 2006; Gal-Yam et al. 2006; Fynbo et al. 2006; Della Valle et al. 2006), and short GRBs that are likely associated with massive stars (e.g. GRB 090426, Levesque et al. 2010; Antonelli et al. 2009; Xin et al. 2011; Thöne et al. 2011). Zhang et al. (2007b) and Zhang (2006) proposed the Type I vs. Type II classification scheme in parallel with the short vs. long scheme, and Zhang et al. (2009) elaborated on the multiple observational criteria to identify the physical category of a certain GRB. Observationally, Type I and Type II GRBs show overlapping behaviors in all individual observational criteria (Li et al.|2016b), suggesting that multi-criteria are needed to perform classification. Zhang et al. (2009) proposed a flowchart to apply multi-criteria to classify GRBs, which was successfully applied to GRBs before 2011 (Kann et al. 2010, 2011).

- Successful vs. choked jets: Within long (Type II) GRBs, bursts may be further classified into successful and choked jets. The former apply to the traditional HL-GRBs, while the latter may apply to some LL-GRBs such as GRB 060218 (e.g. Nakar and Sari 2012; Bromberg et al. 2012).

- Different progenitor stars within Type II GRBs: For GRBs related to massive stars, there might exist different progenitor star systems. The leading type of progenitor star is Wolf-Rayet stars, rapidly rotating massive stars with hydrogen and helium envelope lost before core collapse (Wooslev 1993; MacFadven and Wooslev 1999). The association of long GRBs with Type Ic SNe (no H and He lines) is consistent with such a progenitor picture. Ultra-long GRBs, on the other hand, may call for a different progenitor star system with a larger size, e.g. blue supergiants (Mészáros and Rees 2001; Kashivama et al. 2013; Greiner et al. 2015), but a strong case of a new type of progenitor is not established. Besides single star collapsars (Wooslev and Bloom 2006), a variety of binary progenitor star systems have been also discussed (Frver et al. 1999; Ruffini et al. 2016). No smoking-gun signature has been identified to judge whether long GRB progenitors are in single or binary star systems. 
- Different progenitor stars within Type I GRBs: For GRBs not related to massive stars, the leading models invoke mergers of neutron star (NS) binaries (Paczynski 1986; Eichler et al. 1989; Paczynski 1991; Narayan et al. 1992; Rosswog et al. 2003; Rezzolla et al. 2011), either two NSs (NS-NS), or a NS and a black hole (NS-BH). Other types of progenitors (e.g. accretion-induced-collapse, AIC, of NSs, Qin et al. 1998; Dermer and Atovan 2006) are also possible. A mergerorigin of short GRBs may be verified in the future when gravitational wave chirp signals are discovered to be associated with short GRBs. It is interesting to mention the putative short GRB signal detected with Fermi/GBM that lagged behind the first gravitational wave event GW 150914 by $0.4 \mathrm{~s}$ (Connaughton et al. 2016). If this is the case, BH-BH mergers may also make short GRBs, which may require unconventional mechanisms to produce short GRBs (e.g. Zhang 2016; Loeb 2016; Perna et al.2016; Li et al.2016a; Murase et al. 2016; Yamazaki et al. 2016; Veres et al. 2016).

- GRBs with different central engines: For both long and short GRBs, two types of central engines have been widely discussed in the literature: hyper-accreting black holes (Narayan et al. 1992; Wooslev 1993; Popham et al. 1999) and rapidly rotating magnetars (Usov 1992; Dai and Lu 1998a, b;; Zhang and Mészáros 2001; Metzger et al. 2011). Signatures of magnetar spindown have been seen in both long and short GRBs (Lü and Zhang 2014; Lü et al. 2015, and references therein). In particular, a possible magnetar central engine for short GRBs (Dai et al. 2006; Gao and Fan 2006; Fan and Xu 2006; Metzger et al. 2008), if verified by future gravitational wave data, would have profound implications in constraining poorly known neutron star equation of state (Lasky et al. 2014; Lü et al. 2015; Gao et al. 2016; Li et al. 2016) and in searching for electromagnetic counterparts of gravitational wave events (Zhang 2013; Gao et al. 2013b; Yu et al. 2013; Gao et al. 2016).

- Other bursts no longer defined as GRBs: Historically several phenomena were initially confused as GRBs and later found to be other distinct phenomena that also emit bursts of $\gamma$-rays. An early example was the so-called soft-gamma-ray repeaters (SGRs), now known as bursts from Galactic magnetars. Some SGRBs emit giant flares characterized with a short hard spike followed by oscillating X-ray pulses (e.g. Palmer et al. 2005). The short hard spikes of SGR giant flares can be detectable up to $80 \mathrm{Mpc}$, which may be confused as short GRBs. A systematic search suggested that these giant-flare-origin short GRBs may comprise less than $5 \%$ of the short GRB population (Tanvir et al. 2005). One candidate of such events, GRB 051103, has been discovered (Frederiks et al. 2007). Another example was "GRB 110328" which was later identified as a tidal disruption event that launched a jet beaming towards earth (Bloom et al. 2011; Burrows et al. 2011) and renamed as Sw 1644+57. Finally, it has been long proposed that evaporation of primordial black holes with mass $\sim 10^{17}$ g (Hawking 1975) may be able to produce ultra-short GRBs. However, no robust evidence for the existence of these bursts has been collected so far (Ukwatta et al. 2016, and references therein). 


\section{Summary}

After nearly half century of observations, rich data have been accumulated for GRB prompt emission and afterglow. Our physical understanding of GRBs, including their progenitor systems, central engines, jet properties (composition, Lorentz factor), energy dissipation and radiation mechanisms, as well as the interaction between jet and ambient medium, has been greatly advanced. Yet, many open questions remain, which call for more dedicated multi-wavelength, multi-messenger observations that cover the full temporal and spectral windows in the prompt emission and afterglow phases.

Acknowledgements BZ acknowledges the NASA ADAP (NNX14AF85G) and ATP (NNX15AK85G) programs for supports. The research at Guangxi University was supported by the National Basic Research Program (973 Program) of China 2014CB845800, the National Natural Science Foundation of China (Grant No. 11533003,11603006,U1331202), One-Hundred-Talents Program of Guangxi colleges, Scientific Research Foundation of GuangXi University (Grant No XGZ150299), and Guangxi Science Foundation (grant No. 2013GXNSFFA019001,2016GXNSFCB380005). 

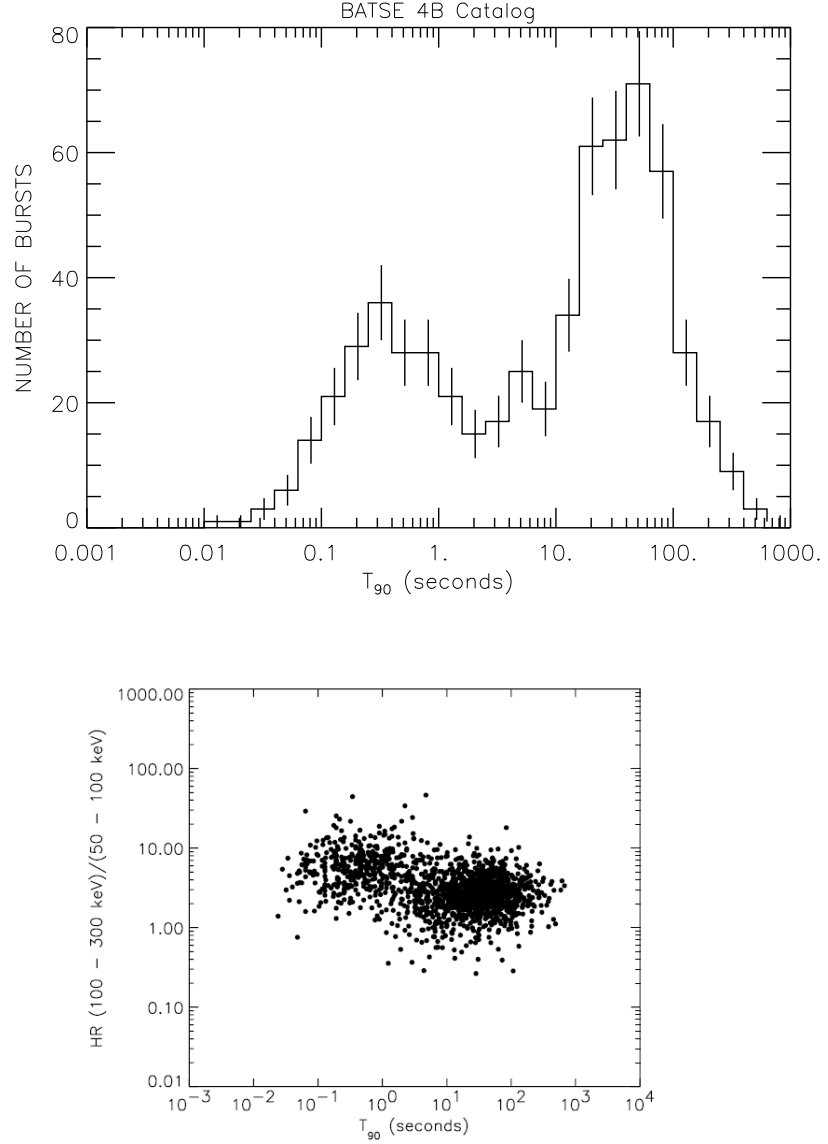

Fig. 1 Left: The bimodal distribution of GRB durations. Right: The two dimensional distribution of GRBs in the $T_{90}-\mathrm{HR}$ domain. From BATSE GRB catalogs (http://gammaray.msfc.nasa.gov/batse/grb/catalog/). 

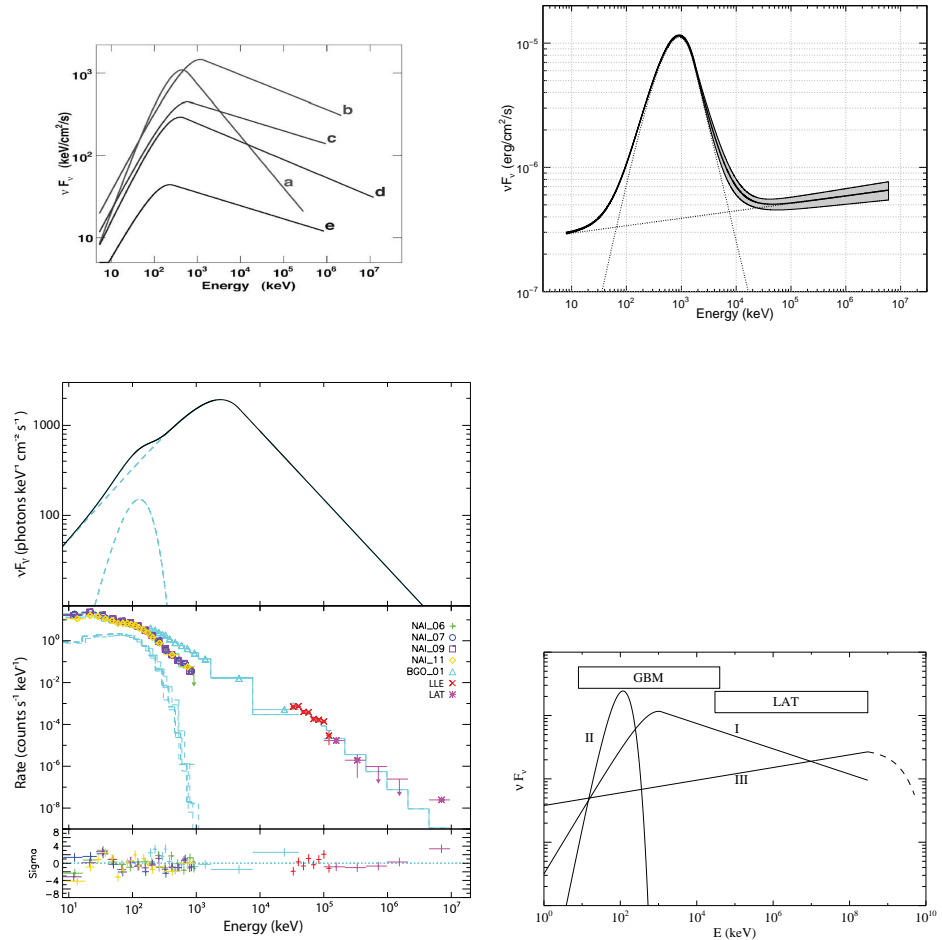

Fig. 2 Some example GRB spectra: (a) the Band function in GRB 080916c Abdo et al. 2009b); (b) a quasi-thermal spectrum superposed on a power law component in GRB 090902B (Abdo et al. 2009a); (c) a thermal component superposed on a Band component in GRB 110721 (Axelsson et al. 2012); (d) three possible elemental spectral components that shape GRB spectra (Zhang et al. 2011). 

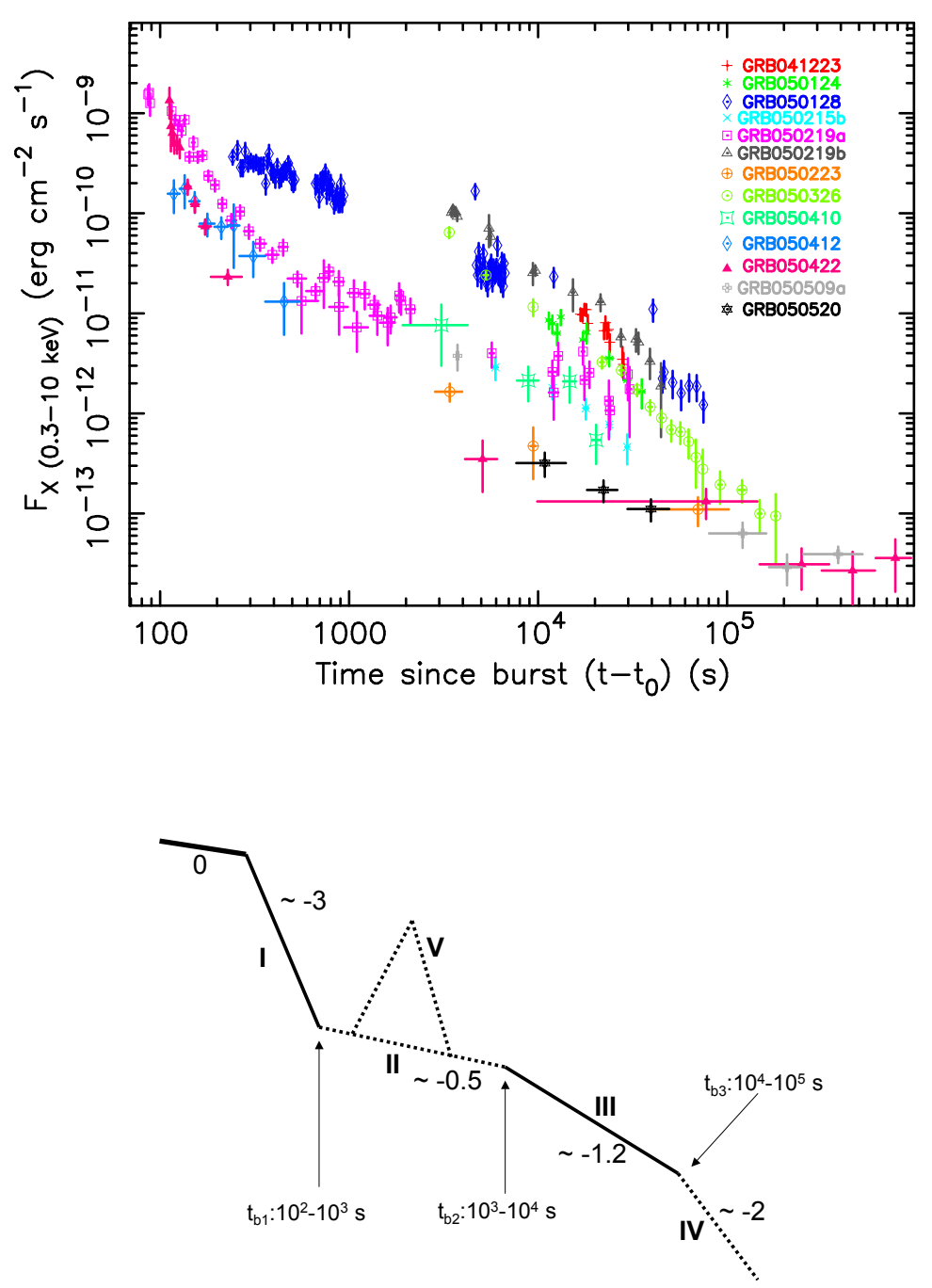

Fig. 3 Left: Examples of Observed XRT lightcurves (Nousek et al. 2006). Right: A canonical XRT lightcurve (Zhang et al. 2006). 

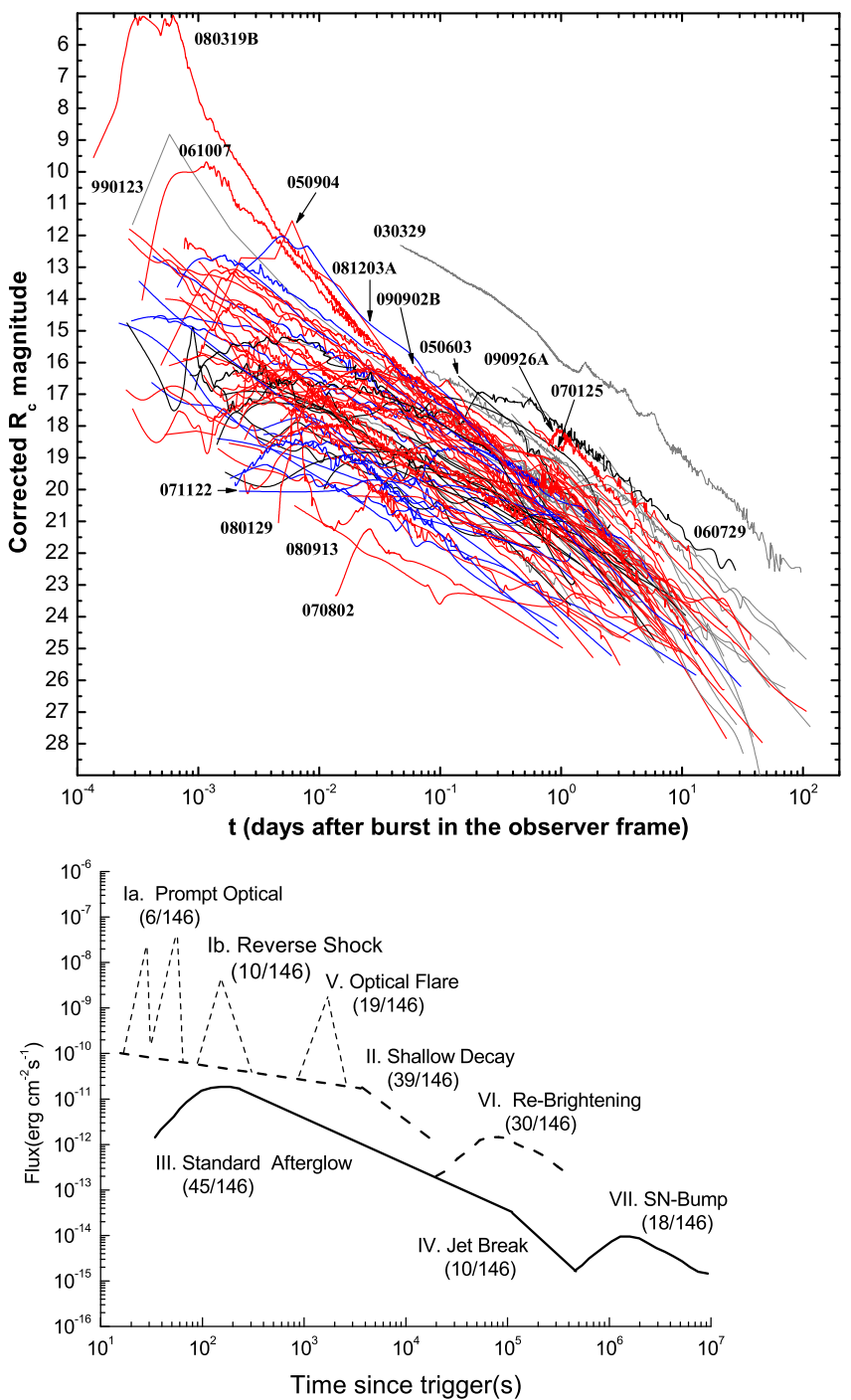

Fig. 4 (a) Observer-frame optical lightcurves for a sample of GRBs (Kann et al. 2010); (2) Synthetic schematic light curve of multiple optical emission components, from ( $\mathrm{Li}$ et al. 2012). 

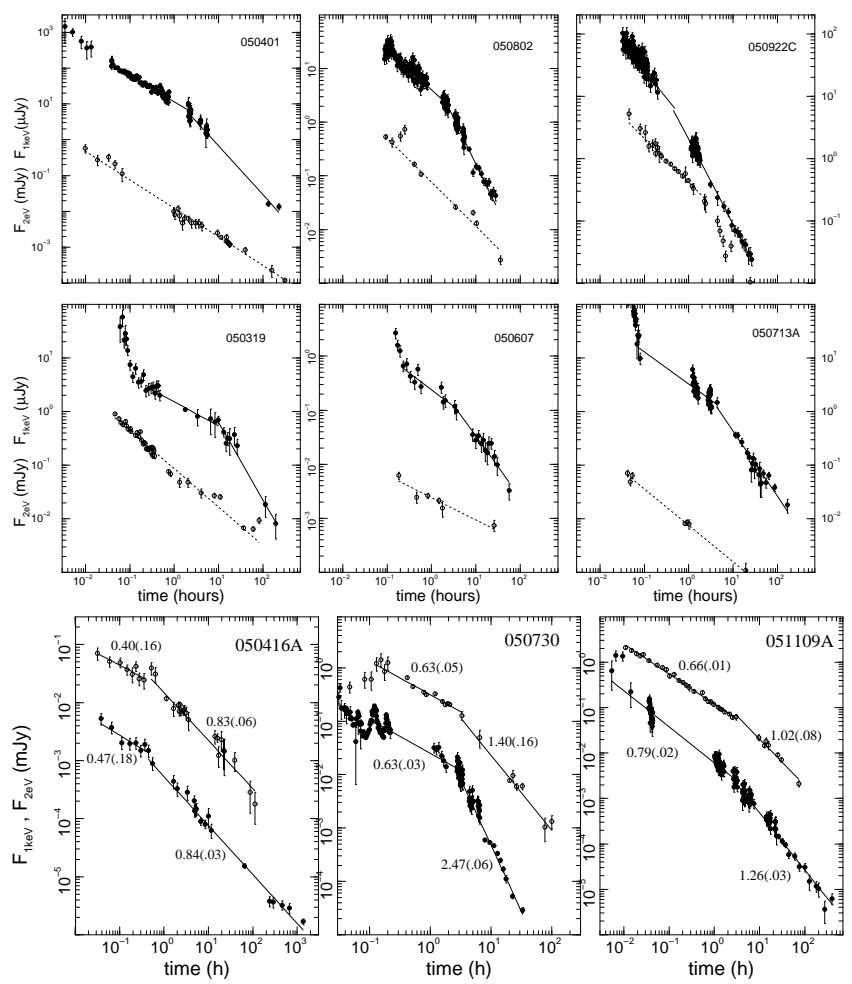

Fig. 5 Examples of chromatic and achromatic afterglow lightcurves, from (Panaitescu et al. 2006).

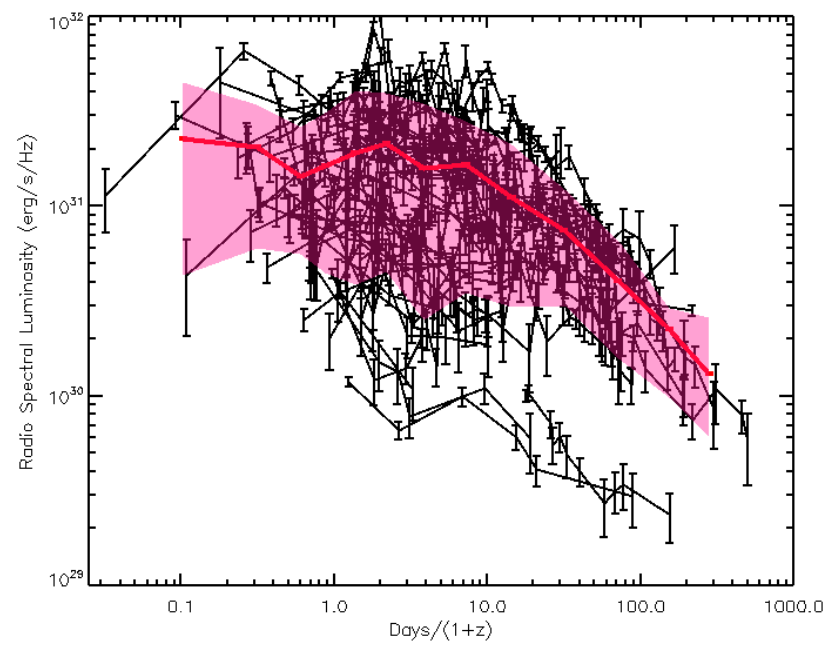

Fig. 6 The radio light curves at $8.5 \mathrm{GHz}$ for the long GRBs in the rest frame time. The red solid line represents the mean light curve. The pink shaded area represents the $75 \%$ confidence band, from Chandra and Frail (2012). 


\section{References}

M.G. Aartsen, K. Abraham, M. Ackermann, J. Adams, J.A. Aguilar, M. Ahlers, M. Ahrens, D. Altmann, T. Anderson, I. Ansseau, et al., An All-sky Search for Three Flavors of Neutrinos from Gamma-ray Bursts with the IceCube Neutrino Observatory. Astrophys. J. 824, 115 (2016). doi:10.3847/0004-637X/824/2/115

R. Abbasi, Y. Abdou, T. Abu-Zayyad, M. Ackermann, J. Adams, J.A. Aguilar, M. Ahlers, D. Altmann, K. Andeen, J. Auffenberg, et al., An absence of neutrinos associated with cosmicray acceleration in $\gamma$-ray bursts. Natur. 484, 351-354 (2012). doi:10.1038/nature11068

A.A. Abdo, M. Ackermann, M. Ajello, K. Asano, W.B. Atwood, M. Axelsson, L. Baldini, J. Ballet, G. Barbiellini, M.G. Baring, D. Bastieri, K. Bechtol, R. Bellazzini, B. Berenji, P.N. Bhat, E. Bissaldi, R.D. Blandford, E.D. Bloom, E. Bonamente, A.W. Borgland, A. Bouvier, J. Bregeon, A. Brez, M.S. Briggs, M. Brigida, P. Bruel, J.M. Burgess, D.N. Burrows, S. Buson, G.A. Caliandro, R.A. Cameron, P.A. Caraveo, J.M. Casandjian, C. Cecchi, Ö. Celik, A. Chekhtman, C.C. Cheung, J. Chiang, S. Ciprini, R. Claus, J. Cohen-Tanugi, L.R. Cominsky, V. Connaughton, J. Conrad, S. Cutini, V. d'Elia, C.D. Dermer, A. de Angelis, F. de Palma, S.W. Digel, B.L. Dingus, E.d.C.e. Silva, P.S. Drell, R. Dubois, D. Dumora, C. Farnier, C. Favuzzi, S.J. Fegan, J. Finke, G. Fishman, W.B. Focke, P. Fortin, M. Frailis, Y. Fukazawa, S. Funk, P. Fusco, F. Gargano, N. Gehrels, S. Germani, G. Giavitto, B. Giebels, N. Giglietto, F. Giordano, T. Glanzman, G. Godfrey, A. Goldstein, J. Granot, J. Greiner, I.A. Grenier, J.E. Grove, L. Guillemot, S. Guiriec, Y. Hanabata, A.K. Harding, M. Hayashida, E. Hays, D. Horan, R.E. Hughes, M.S. Jackson, G. Jóhannesson, A.S. Johnson, R.P. Johnson, W.N. Johnson, T. Kamae, H. Katagiri, J. Kataoka, N. Kawai, M. Kerr, R.M. Kippen, J. Knödlseder, D. Kocevski, N. Komin, C. Kouveliotou, M. Kuss, J. Lande, L. Latronico, M. Lemoine-Goumard, F. Longo, F. Loparco, B. Lott, M.N. Lovellette, P. Lubrano, G.M. Madejski, A. Makeev, M.N. Mazziotta, S. McBreen, J.E. McEnery, S. McGlynn, C. Meegan, P. Mészáros, C. Meurer, P.F. Michelson, W. Mitthumsiri, T. Mizuno, A.A. Moiseev, C. Monte, M.E. Monzani, E. Moretti, A. Morselli, I.V. Moskalenko, S. Murgia, T. Nakamori, P.L. Nolan, J.P. Norris, E. Nuss, M. Ohno, T. Ohsugi, N. Omodei, E. Orlando, J.F. Ormes, W.S. Paciesas, D. Paneque, J.H. Panetta, V. Pelassa, M. Pepe, M. Pesce-Rollins, V. Petrosian, F. Piron, T.A. Porter, R. Preece, S. Rainò, R. Rando, A. Rau, M. Razzano, S. Razzaque, A. Reimer, O. Reimer, T. Reposeur, S. Ritz, L.S. Rochester, A.Y. Rodriguez, P.W.A. Roming, M. Roth, F. Ryde, H.F.-W. Sadrozinski, D. Sanchez, A. Sander, P.M. Saz Parkinson, J.D. Scargle, T.L. Schalk, C. Sgrò, E.J. Siskind, P.D. Smith, P. Spinelli, M. Stamatikos, F.W. Stecker, G. Stratta, M.S. Strickman, D.J. Suson, C.A. Swenson, H. Tajima, H. Takahashi, T. Tanaka, J.B. Thayer, J.G. Thayer, D.J. Thompson, L. Tibaldo, D.F. Torres, G. Tosti, A. Tramacere, Y. Uchiyama, T. Uehara, T.L. Usher, A.J. van der Horst, V. Vasileiou, N. Vilchez, V. Vitale, A. von Kienlin, A.P. Waite, P. Wang, C. Wilson-Hodge, B.L. Winer, K.S. Wood, R. Yamazaki, T. Ylinen, M. Ziegler, Fermi Observations of GRB 090902B: A Distinct Spectral Component in the Prompt and Delayed Emission. Astrophys. J. Lett. 706, 138-144 (2009a). doi:10.1088/0004-637X/706/1/L138

A.A. Abdo, M. Ackermann, M. Arimoto, K. Asano, W.B. Atwood, M. Axelsson, L. Baldini, J. Ballet, D.L. Band, G. Barbiellini, et al., Fermi Observations of High-Energy Gamma-Ray Emission from GRB 080916C. Science 323, 1688 (2009b). doi:10.1126/science.1169101

M. Ackermann, K. Asano, W.B. Atwood, M. Axelsson, L. Baldini, J. Ballet, G. Barbiellini, M.G. Baring, D. Bastieri, K. Bechtol, R. Bellazzini, B. Berenji, P.N. Bhat, E. Bissaldi, R.D. Blandford, E.D. Bloom, E. Bonamente, A.W. Borgland, A. Bouvier, J. Bregeon, A. Brez, M.S. Briggs, M. Brigida, P. Bruel, S. Buson, G.A. Caliandro, R.A. Cameron, P.A. Caraveo, S. Carrigan, J.M. Casandjian, C. Cecchi, Ö. Celik, E. Charles, J. Chiang, S. Ciprini, R. Claus, J. Cohen-Tanugi, V. Connaughton, J. Conrad, C.D. Dermer, F. de Palma, B.L. Dingus, E.d.C.e. Silva, P.S. Drell, R. Dubois, D. Dumora, C. Farnier, C. Favuzzi, S.J. Fegan, J. Finke, W.B. Focke, M. Frailis, Y. Fukazawa, P. Fusco, F. Gargano, D. Gasparrini, N. Gehrels, S. Germani, N. Giglietto, F. Giordano, T. Glanzman, G. Godfrey, J. Granot, I.A. Grenier, M.-H. Grondin, J.E. Grove, S. Guiriec, D. Hadasch, A.K. Harding, E. Hays, D. Horan, R.E. Hughes, G. Jóhannesson, W.N. Johnson, T. Kamae, H. Katagiri, J. Kataoka, N. Kawai, R.M. Kippen, J. Knödlseder, D. Kocevski, C. Kouveliotou, M. Kuss, J. Lande, L. Latronico, M. Lemoine-Goumard, M. Llena Garde, F. Longo, F. Loparco, B. Lott, M.N. Lovellette, P. Lubrano, A. Makeev, M.N. Mazziotta, J.E. McEnery, S. McGlynn, C. Meegan, P. Mészáros, P.F. Michelson, W. Mitthumsiri, T. Mizuno, A.A. Moiseev, C. Monte, M.E. Monzani, E. Moretti, A. Morselli, I.V. Moskalenko, S. Murgia, H. Naka- 
jima, T. Nakamori, P.L. Nolan, J.P. Norris, E. Nuss, M. Ohno, T. Ohsugi, N. Omodei, E. Orlando, J.F. Ormes, M. Ozaki, W.S. Paciesas, D. Paneque, J.H. Panetta, D. Parent, V. Pelassa, M. Pepe, M. Pesce-Rollins, F. Piron, R. Preece, S. Rainò, R. Rando, M. Razzano, S. Razzaque, A. Reimer, S. Ritz, A.Y. Rodriguez, M. Roth, F. Ryde, H.F.-W. Sadrozinski, A. Sander, J.D. Scargle, T.L. Schalk, C. Sgrò, E.J. Siskind, P.D. Smith, G. Spandre, P. Spinelli, M. Stamatikos, F.W. Stecker, M.S. Strickman, D.J. Suson, H. Tajima, H. Takahashi, T. Takahashi, T. Tanaka, J.B. Thayer, J.G. Thayer, D.J. Thompson, L. Tibaldo, K. Toma, D.F. Torres, G. Tosti, A. Tramacere, Y. Uchiyama, T. Uehara, T.L. Usher, A.J. van der Horst, V. Vasileiou, N. Vilchez, V. Vitale, A. von Kienlin, A.P. Waite, P. Wang, C. Wilson-Hodge, B.L. Winer, X.F. Wu, R. Yamazaki, Z. Yang, T. Ylinen, M. Ziegler, Fermi Observations of GRB 090510: A Short-Hard Gamma-ray Burst with an Additional, Hard Power-law Component from $10 \mathrm{keV}$ TO GeV Energies. Astrophys. J. 716, 1178-1190 (2010). doi:10.1088/0004-637X/716/2/1178

M. Ackermann, M. Ajello, K. Asano, M. Axelsson, L. Baldini, J. Ballet, G. Barbiellini, M.G. Baring, D. Bastieri, K. Bechtol, R. Bellazzini, B. Berenji, P.N. Bhat, E. Bissaldi, R.D. Blandford, E. Bonamente, A.W. Borgland, A. Bouvier, J. Bregeon, A. Brez, M.S. Briggs, M. Brigida, P. Bruel, R. Buehler, S. Buson, G.A. Caliandro, R.A. Cameron, P.A. Caraveo, S. Carrigan, J.M. Casandjian, C. Cecchi, Ö. Çelik, V. Chaplin, E. Charles, A. Chekhtman, J. Chiang, S. Ciprini, R. Claus, J. Cohen-Tanugi, V. Connaughton, J. Conrad, S. Cutini, C.D. Dermer, A. de Angelis, F. de Palma, B.L. Dingus, E.d.C.e. Silva, P.S. Drell, R. Dubois, C. Favuzzi, S.J. Fegan, E.C. Ferrara, W.B. Focke, M. Frailis, Y. Fukazawa, S. Funk, P. Fusco, F. Gargano, D. Gasparrini, N. Gehrels, S. Germani, N. Giglietto, F. Giordano, M. Giroletti, T. Glanzman, G. Godfrey, A. Goldstein, J. Granot, J. Greiner, I.A. Grenier, J.E. Grove, S. Guiriec, D. Hadasch, Y. Hanabata, A.K. Harding, K. Hayashi, M. Hayashida, E. Hays, D. Horan, R.E. Hughes, R. Itoh, G. Jóhannesson, A.S. Johnson, W.N. Johnson, T. Kamae, H. Katagiri, J. Kataoka, R.M. Kippen, J. Knödlseder, D. Kocevski, C. Kouveliotou, M. Kuss, J. Lande, L. Latronico, S.-H. Lee, M. Llena Garde, F. Longo, F. Loparco, M.N. Lovellette, P. Lubrano, A. Makeev, M.N. Mazziotta, S. McBreen, J.E. McEnery, S. McGlynn, C. Meegan, J. Mehault, P. Mészáros, P.F. Michelson, T. Mizuno, C. Monte, M.E. Monzani, E. Moretti, A. Morselli, I.V. Moskalenko, S. Murgia, H. Nakajima, T. Nakamori, M. Naumann-Godo, S. Nishino, P.L. Nolan, J.P. Norris, E. Nuss, M. Ohno, T. Ohsugi, A. Okumura, N. Omodei, E. Orlando, J.F. Ormes, M. Ozaki, W.S. Paciesas, D. Paneque, J.H. Panetta, D. Parent, V. Pelassa, M. Pepe, M. Pesce-Rollins, V. Petrosian, F. Piron, T.A. Porter, R. Preece, J.L. Racusin, S. Rainò, R. Rando, A. Rau, M. Razzano, S. Razzaque, A. Reimer, O. Reimer, T. Reposeur, L.C. Reyes, J. Ripken, S. Ritz, M. Roth, F. Ryde, H.F.-W. Sadrozinski, A. Sander, J.D. Scargle, T.L. Schalk, C. Sgrò, E.J. Siskind, P.D. Smith, G. Spandre, P. Spinelli, M. Stamatikos, F.W. Stecker, M.S. Strickman, D.J. Suson, H. Tajima, H. Takahashi, T. Tanaka, Y. Tanaka, J.B. Thayer, J.G. Thayer, L. Tibaldo, D. Tierney, K. Toma, D.F. Torres, G. Tosti, A. Tramacere, Y. Uchiyama, T. Uehara, T.L. Usher, J. Vandenbroucke, A.J. van der Horst, V. Vasileiou, N. Vilchez, V. Vitale, A. von Kienlin, A.P. Waite, P. Wang, C. Wilson-Hodge, B.L. Winer, K.S. Wood, X.F. Wu, R. Yamazaki, Z. Yang, T. Ylinen, M. Ziegler, Detection of a Spectral Break in the Extra Hard Component of GRB 090926A. Astrophys. J. 729, 114 (2011). doi:10.1088/0004-637X/729/2/114

M. Ackermann, M. Ajello, K. Asano, L. Baldini, G. Barbiellini, M.G. Baring, D. Bastieri, R. Bellazzini, R.D. Blandford, E. Bonamente, A.W. Borgland, E. Bottacini, J. Bregeon, M. Brigida, P. Bruel, R. Buehler, S. Buson, G.A. Caliandro, R.A. Cameron, P.A. Caraveo, C. Cecchi, E. Charles, R.C.G. Chaves, A. Chekhtman, J. Chiang, S. Ciprini, R. Claus, J. Cohen-Tanugi, J. Conrad, S. Cutini, F. D'Ammando, A. de Angelis, F. de Palma, C.D. Dermer, E.d.C.e. Silva, P.S. Drell, A. Drlica-Wagner, C. Favuzzi, S.J. Fegan, W.B. Focke, A. Franckowiak, Y. Fukazawa, P. Fusco, F. Gargano, D. Gasparrini, N. Gehrels, N. Giglietto, F. Giordano, M. Giroletti, T. Glanzman, G. Godfrey, J. Granot, J. Greiner, I.A. Grenier, J.E. Grove, S. Guiriec, D. Hadasch, Y. Hanabata, M. Hayashida, E. Hays, R.E. Hughes, M.S. Jackson, T. Jogler, G. Jóhannesson, A.S. Johnson, J. Knödlseder, D. Kocevski, M. Kuss, J. Lande, S. Larsson, L. Latronico, F. Longo, F. Loparco, M.N. Lovellette, P. Lubrano, M.N. Mazziotta, J.E. McEnery, J. Mehault, P. Mészáros, P.F. Michelson, W. Mitthumsiri, T. Mizuno, C. Monte, M.E. Monzani, E. Moretti, A. Morselli, I.V. Moskalenko, S. Murgia, M. Naumann-Godo, J.P. Norris, E. Nuss, T. Nymark, M. Ohno, T. Ohsugi, N. Omodei, M. Orienti, E. Orlando, D. Paneque, J.S. Perkins, M. Pesce-Rollins, F. Piron, G. Pivato, J.L. Racusin, S. Rainò, R. Rando, M. Razzano, S. Razzaque, A. Reimer, 
O. Reimer, C. Romoli, M. Roth, F. Ryde, D.A. Sanchez, C. Sgrò, E.J. Siskind, E. Sonbas, P. Spinelli, M. Stamatikos, H. Takahashi, T. Tanaka, J.G. Thayer, J.B. Thayer, L. Tibaldo, M. Tinivella, G. Tosti, E. Troja, T.L. Usher, J. Vandenbroucke, V. Vasileiou, G. Vianello, V. Vitale, A.P. Waite, B.L. Winer, K.S. Wood, Z. Yang, D. Gruber, P.N. Bhat, E. Bissaldi, M.S. Briggs, J.M. Burgess, V. Connaughton, S. Foley, R.M. Kippen, C. Kouveliotou, S. McBreen, S. McGlynn, W.S. Paciesas, V. Pelassa, R. Preece, A. Rau, A.J. van der Horst, A. von Kienlin, D.A. Kann, R. Filgas, S. Klose, T. Krühler, A. Fukui, T. Sako, P.J. Tristram, S.R. Oates, T.N. Ukwatta, O. Littlejohns, Multiwavelength Observations of GRB 110731A: GeV Emission from Onset to Afterglow. Astrophys. J. 763, 71 (2013). doi:10.1088/0004-637X/763/2/71

M. Ackermann, M. Ajello, K. Asano, W.B. Atwood, M. Axelsson, L. Baldini, J. Ballet, G. Barbiellini, M.G. Baring, D. Bastieri, K. Bechtol, R. Bellazzini, E. Bissaldi, E. Bonamente, J. Bregeon, M. Brigida, P. Bruel, R. Buehler, J.M. Burgess, S. Buson, G.A. Caliandro, R.A. Cameron, P.A. Caraveo, C. Cecchi, V. Chaplin, E. Charles, A. Chekhtman, C.C. Cheung, J. Chiang, G. Chiaro, S. Ciprini, R. Claus, W. Cleveland, J. Cohen-Tanugi, A. Collazzi, L.R. Cominsky, V. Connaughton, J. Conrad, S. Cutini, F. D'Ammando, A. de Angelis, M. DeKlotz, F. de Palma, C.D. Dermer, R. Desiante, A. Diekmann, L. Di Venere, P.S. Drell, A. Drlica-Wagner, C. Favuzzi, S.J. Fegan, E.C. Ferrara, J. Finke, G. Fitzpatrick, W.B. Focke, A. Franckowiak, Y. Fukazawa, S. Funk, P. Fusco, F. Gargano, N. Gehrels, S. Germani, M. Gibby, N. Giglietto, M. Giles, F. Giordano, M. Giroletti, G. Godfrey, J. Granot, I.A. Grenier, J.E. Grove, D. Gruber, S. Guiriec, D. Hadasch, Y. Hanabata, A.K. Harding, M. Hayashida, E. Hays, D. Horan, R.E. Hughes, Y. Inoue, T. Jogler, G. Jóhannesson, W.N. Johnson, T. Kawano, J. Knödlseder, D. Kocevski, M. Kuss, J. Lande, S. Larsson, L. Latronico, F. Longo, F. Loparco, M.N. Lovellette, P. Lubrano, M. Mayer, M.N. Mazziotta, J.E. McEnery, P.F. Michelson, T. Mizuno, A.A. Moiseev, M.E. Monzani, E. Moretti, A. Morselli, I.V. Moskalenko, S. Murgia, R. Nemmen, E. Nuss, M. Ohno, T. Ohsugi, A. Okumura, N. Omodei, M. Orienti, D. Paneque, V. Pelassa, J.S. Perkins, M. Pesce-Rollins, V. Petrosian, F. Piron, G. Pivato, T.A. Porter, J.L. Racusin, S. Rainò, R. Rando, M. Razzano, S. Razzaque, A. Reimer, O. Reimer, S. Ritz, M. Roth, F. Ryde, A. Sartori, P.M.S. Parkinson, J.D. Scargle, A. Schulz, C. Sgrò, E.J. Siskind, E. Sonbas, G. Spandre, P. Spinelli, H. Tajima, H. Takahashi, J.G. Thayer, J.B. Thayer, D.J. Thompson, L. Tibaldo, M. Tinivella, D.F. Torres, G. Tosti, E. Troja, T.L. Usher, J. Vandenbroucke, V. Vasileiou, G. Vianello, V. Vitale, B.L. Winer, K.S. Wood, R. Yamazaki, G. Younes, H.-F. Yu, S.J. Zhu, P.N. Bhat, M.S. Briggs, D. Byrne, S. Foley, A. Goldstein, P. Jenke, R.M. Kippen, C. Kouveliotou, S. McBreen, C. Meegan, W.S. Paciesas, R. Preece, A. Rau, D. Tierney, A.J. van der Horst, A. von Kienlin, C. Wilson-Hodge, S. Xiong, G. Cusumano, V. La Parola, J.R. Cummings, Fermi-LAT Observations of the Gamma-Ray Burst GRB 130427A. Science 343, 42-47 (2014). doi:10.1126/science.1242353

C. Akerlof, R. Balsano, S. Barthelmy, J. Bloch, P. Butterworth, D. Casperson, T. Cline, S. Fletcher, F. Frontera, G. Gisler, J. Heise, J. Hills, R. Kehoe, B. Lee, S. Marshall, T. McKay, R. Miller, L. Piro, W. Priedhorsky, J. Szymanski, J. Wren, Observation of contemporaneous optical radiation from a $\gamma$-ray burst. Natur. 398, 400-402 (1999). doi:10.1038/18837

G.E. Anderson, A.J. van der Horst, T.D. Staley, R.P. Fender, R.A.M.J. Wijers, A.M.M. Scaife, C. Rumsey, D.J. Titterington, A. Rowlinson, R.D.E. Saunders, Probing the bright radio flare and afterglow of GRB 130427A with the Arcminute Microkelvin Imager. Mon. Not. Roy. Astron. Soc. 440, 2059-2065 (2014). doi:10.1093/mnras/stu478

L.A. Antonelli, P. D'Avanzo, R. Perna, L. Amati, S. Covino, S. Cutini, V. D'Elia, S. Gallozzi, A. Grazian, E. Palazzi, S. Piranomonte, A. Rossi, S. Spiro, L. Stella, V. Testa, G. Chincarini, A. di Paola, F. Fiore, D. Fugazza, E. Giallongo, E. Maiorano, N. Masetti, F. Pedichini, R. Salvaterra, G. Tagliaferri, S. Vergani, GRB 090426: the farthest short gamma-ray burst? Astron. Astrophys. 507, 45-48 (2009). doi:10.1051/0004-6361/200913062

M. Axelsson, L. Baldini, G. Barbiellini, M.G. Baring, R. Bellazzini, J. Bregeon, M. Brigida, P. Bruel, R. Buehler, G.A. Caliandro, R.A. Cameron, P.A. Caraveo, C. Cecchi, R.C.G. Chaves, A. Chekhtman, J. Chiang, R. Claus, J. Conrad, S. Cutini, F. D'Ammando, F. de Palma, C.D. Dermer, E.d.C.e. Silva, P.S. Drell, C. Favuzzi, S.J. Fegan, E.C. Ferrara, W.B. Focke, Y. Fukazawa, P. Fusco, F. Gargano, D. Gasparrini, N. Gehrels, S. Germani, N. Giglietto, M. Giroletti, G. Godfrey, S. Guiriec, D. Hadasch, Y. Hanabata, M. Hayashida, X. Hou, S. Iyyani, M.S. Jackson, D. Kocevski, M. Kuss, J. Larsson, S. Larsson, F. Longo, F. Loparco, C. Lundman, M.N. Mazziotta, J.E. McEnery, T. Mizuno, M.E. Monzani, E. Moretti, A. Morselli, S. Murgia, E. Nuss, T. Nymark, M. Ohno, N. Omodei, M. Pesce- 
Rollins, F. Piron, G. Pivato, J.L. Racusin, S. Rainò, M. Razzano, S. Razzaque, A. Reimer, M. Roth, F. Ryde, D.A. Sanchez, C. Sgrò, E.J. Siskind, G. Spandre, P. Spinelli, M. Stamatikos, L. Tibaldo, M. Tinivella, T.L. Usher, J. Vandenbroucke, V. Vasileiou, G. Vianello, V. Vitale, A.P. Waite, B.L. Winer, K.S. Wood, J.M. Burgess, P.N. Bhat, E. Bissaldi, M.S. Briggs, V. Connaughton, G. Fishman, G. Fitzpatrick, S. Foley, D. Gruber, R.M. Kippen, C. Kouveliotou, P. Jenke, S. McBreen, S. McGlynn, C. Meegan, W.S. Paciesas, V. Pelassa, R. Preece, D. Tierney, A. von Kienlin, C. Wilson-Hodge, S. Xiong, A. Pe'er, GRB110721A: An Extreme Peak Energy and Signatures of the Photosphere. Astrophys. J. Lett. 757, 31 (2012). doi:10.1088/2041-8205/757/2/L31

D. Band, J. Matteson, L. Ford, B. Schaefer, D. Palmer, B. Teegarden, T. Cline, M. Briggs, W. Paciesas, G. Pendleton, G. Fishman, C. Kouveliotou, C. Meegan, R. Wilson, P. Lestrade, BATSE observations of gamma-ray burst spectra. I - Spectral diversity. Astrophys. J. 413, 281-292 (1993). doi:10.1086/172995

S.D. Barthelmy, J.K. Cannizzo, N. Gehrels, G. Cusumano, V. Mangano, P.T. O’Brien, S. Vaughan, B. Zhang, D.N. Burrows, S. Campana, G. Chincarini, M.R. Goad, C. Kouveliotou, P. Kumar, P. Mészáros, J.A. Nousek, J.P. Osborne, A. Panaitescu, J.N. Reeves, T. Sakamoto, G. Tagliaferri, R.A.M.J. Wijers, Discovery of an Afterglow Extension of the Prompt Phase of Two Gamma-Ray Bursts Observed by Swift. Astrophys. J. Lett. 635, 133-136 (2005). doi:10.1086/499432

A.M. Beloborodov, Collisional mechanism for gamma-ray burst emission. Mon. Not. Roy. Astron. Soc. 407, 1033-1047 (2010). doi:10.1111/j.1365-2966.2010.16770.x

A.M. Beloborodov, B.E. Stern, R. Svensson, Power Density Spectra of Gamma-Ray Bursts. Astrophys. J. 535, 158-166 (2000). doi:10.1086/308836

J.S. Bloom, D. Giannios, B.D. Metzger, S.B. Cenko, D.A. Perley, N.R. Butler, N.R. Tanvir, A.J. Levan, P.T. O'Brien, L.E. Strubbe, F. De Colle, E. Ramirez-Ruiz, W.H. Lee, S. Nayakshin, E. Quataert, A.R. King, A. Cucchiara, J. Guillochon, G.C. Bower, A.S. Fruchter, A.N. Morgan, A.J. van der Horst, A Possible Relativistic Jetted Outburst from a Massive Black Hole Fed by a Tidally Disrupted Star. Science 333, 203 (2011). doi:10.1126/science.1207150

M. Boër, B. Gendre, G. Stratta, Are Ultra-long Gamma-Ray Bursts Different? Astrophys. J. 800, 16 (2015). doi:10.1088/0004-637X/800/1/16

O. Bromberg, E. Nakar, T. Piran, R. Sari, An Observational Imprint of the Collapsar Model of Long Gamma-Ray Bursts. Astrophys. J. 749, 110 (2012). doi:10.1088/0004$637 \mathrm{X} / 749 / 2 / 110$

D. Burlon, G. Ghirlanda, G. Ghisellini, D. Lazzati, L. Nava, M. Nardini, A. Celotti, Precursors in Swift Gamma Ray Bursts with Redshift. Astrophys. J. Lett. 685, 19 (2008). doi:10.1086/592350

D.N. Burrows, P. Romano, A. Falcone, S. Kobayashi, B. Zhang, A. Moretti, P.T. O'Brien, M.R. Goad, S. Campana, K.L. Page, L. Angelini, S. Barthelmy, A.P. Beardmore, M. Capalbi, G. Chincarini, J. Cummings, G. Cusumano, D. Fox, P. Giommi, J.E. Hill, J.A. Kennea, H. Krimm, V. Mangano, F. Marshall, P. Mészáros, D.C. Morris, J.A. Nousek, J.P. Osborne, C. Pagani, M. Perri, G. Tagliaferri, A.A. Wells, S. Woosley, N. Gehrels, Bright X-ray Flares in Gamma-Ray Burst Afterglows. Science 309, 1833-1835 (2005). doi:10.1126/science.1116168

D.N. Burrows, J.A. Kennea, G. Ghisellini, V. Mangano, B. Zhang, K.L. Page, M. Eracleous, P. Romano, T. Sakamoto, A.D. Falcone, J.P. Osborne, S. Campana, A.P. Beardmore, A.A. Breeveld, M.M. Chester, R. Corbet, S. Covino, J.R. Cummings, P. D'Avanzo, V. D'Elia, P. Esposito, P.A. Evans, D. Fugazza, J.M. Gelbord, K. Hiroi, S.T. Holland, K.Y. Huang, M. Im, G. Israel, Y. Jeon, Y.-B. Jeon, H.D. Jun, N. Kawai, J.H. Kim, H.A. Krimm, F.E. Marshall, P. Mészáros, H. Negoro, N. Omodei, W.-K. Park, J.S. Perkins, M. Sugizaki, H.-I. Sung, G. Tagliaferri, E. Troja, Y. Ueda, Y. Urata, R. Usui, L.A. Antonelli, S.D. Barthelmy, G. Cusumano, P. Giommi, A. Melandri, M. Perri, J.L. Racusin, B. Sbarufatti, M.H. Siegel, N. Gehrels, Relativistic jet activity from the tidal disruption of a star by a massive black hole. Natur. 476, 421-424 (2011). doi:10.1038/nature10374

N.R. Butler, D. Kocevski, X-Ray Hardness Evolution in GRB Afterglows and Flares: Late-Time GRB Activity without $\mathrm{N}_{H}$ Variations. Astrophys. J. 663, 407-419 (2007). doi:10.1086/518023

S. Campana, V. Mangano, A.J. Blustin, P. Brown, D.N. Burrows, G. Chincarini, J.R. Cummings, G. Cusumano, M. Della Valle, D. Malesani, P. Mészáros, J.A. Nousek, M. Page, T. Sakamoto, E. Waxman, B. Zhang, Z.G. Dai, N. Gehrels, S. Immler, F.E. Marshall, K.O. Mason, A. Moretti, P.T. O'Brien, J.P. Osborne, K.L. Page, P. Romano, P.W.A. 
Roming, G. Tagliaferri, L.R. Cominsky, P. Giommi, O. Godet, J.A. Kennea, H. Krimm, L. Angelini, S.D. Barthelmy, P.T. Boyd, D.M. Palmer, A.A. Wells, N.E. White, The association of GRB 060218 with a supernova and the evolution of the shock wave. Natur. 442, 1008-1010 (2006). doi:10.1038/nature04892

P. Chandra, D.A. Frail, A Radio-selected Sample of Gamma-Ray Burst Afterglows. Astrophys. J. 746, 156 (2012). doi:10.1088/0004-637X/746/2/156

R.A. Chevalier, Z.-Y. Li, Wind Interaction Models for Gamma-Ray Burst Afterglows: The Case for Two Types of Progenitors. Astrophys. J. 536, 195-212 (2000). doi:10.1086/308914

G. Chincarini, A. Moretti, P. Romano, A.D. Falcone, D. Morris, J. Racusin, S. Campana, S. Covino, C. Guidorzi, G. Tagliaferri, D.N. Burrows, C. Pagani, M. Stroh, D. Grupe, M. Capalbi, G. Cusumano, N. Gehrels, P. Giommi, V. La Parola, V. Mangano, T. Mineo, J.A. Nousek, P.T. O'Brien, K.L. Page, M. Perri, E. Troja, R. Willingale, B. Zhang, The First Survey of X-Ray Flares from Gamma-Ray Bursts Observed by Swift: Temporal Properties and Morphology. Astrophys. J. 671, 1903-1920 (2007). doi:10.1086/521591

G. Chincarini, J. Mao, R. Margutti, M.G. Bernardini, C. Guidorzi, F. Pasotti, D. Giannios, M. Della Valle, A. Moretti, P. Romano, P. D'Avanzo, G. Cusumano, P. Giommi, Unveiling the origin of X-ray flares in gamma-ray bursts. Mon. Not. Roy. Astron. Soc. 406, 2113-2148 (2010). doi:10.1111/j.1365-2966.2010.17037.x

V. Connaughton, E. Burns, A. Goldstein, L. Blackburn, M.S. Briggs, B.-B. Zhang, J. Camp, N. Christensen, C.M. Hui, P. Jenke, T. Littenberg, J.E. McEnery, J. Racusin, P. Shawhan, L. Singer, J. Veitch, C.A. Wilson-Hodge, P.N. Bhat, E. Bissaldi, W. Cleveland, G. Fitzpatrick, M.M. Giles, M.H. Gibby, A. von Kienlin, R.M. Kippen, S. McBreen, B. Mailyan, C.A. Meegan, W.S. Paciesas, R.D. Preece, O.J. Roberts, L. Sparke, M. Stanbro, K. Toelge, P. Veres, Fermi GBM Observations of LIGO Gravitational-wave Event GW150914. Astrophys. J. Lett. 826, 6 (2016). doi:10.3847/2041-8205/826/1/L6

E. Costa, F. Frontera, J. Heise, M. Feroci, J. in't Zand, F. Fiore, M.N. Cinti, D. Dal Fiume, L. Nicastro, M. Orlandini, E. Palazzi, M. Rapisarda\#, G. Zavattini, R. Jager, A. Parmar, A. Owens, S. Molendi, G. Cusumano, M.C. Maccarone, S. Giarrusso, A. Coletta, L.A. Antonelli, P. Giommi, J.M. Muller, L. Piro, R.C. Butler, Discovery of an X-ray afterglow associated with the $\gamma$-ray burst of 28 February 1997. Natur. 387, 783-785 (1997). doi: $10.1038 / 42885$

Z.G. Dai, T. Lu, Gamma-ray burst afterglows and evolution of postburst fireballs with energy injection from strongly magnetic millisecond pulsars. Astron. Astrophys. 333, 87-90 (1998a)

Z.G. Dai, T. Lu, Gamma-ray burst afterglows: effects of radiative corrections and nonuniformity of the surrounding medium. Mon. Not. Roy. Astron. Soc. 298, 87-92 (1998b). doi:10.1046/j.1365-8711.1998.01681.x

Z.G. Dai, T. Lu, $\gamma$-Ray Bursts and Afterglows from Rotating Strange Stars and Neutron Stars. Physical Review Letters 81, 4301-4304 (1998c). doi:10.1103/PhysRevLett.81.4301

Z.G. Dai, X.Y. Wang, X.F. Wu, B. Zhang, X-ray Flares from Postmerger Millisecond Pulsars. Science 311, 1127-1129 (2006). doi:10.1126/science.1123606

F. Daigne, R. Mochkovitch, Gamma-ray bursts from internal shocks in a relativistic wind: temporal and spectral properties. Mon. Not. Roy. Astron. Soc. 296, 275-286 (1998). doi:10.1046/j.1365-8711.1998.01305.x

F. Daigne, Ž. Bošnjak, G. Dubus, Reconciling observed gamma-ray burst prompt spectra with synchrotron radiation? Astron. Astrophys. 526, 110 (2011). doi:10.1051/0004$6361 / 201015457$

M.G. Dainotti, R. Willingale, S. Capozziello, V. Fabrizio Cardone, M. Ostrowski, Discovery of a Tight Correlation for Gamma-ray Burst Afterglows with "Canonical" Light Curves. Astrophys. J. Lett. 722, 215-219 (2010). doi:10.1088/2041-8205/722/2/L215

M. de Pasquale, P. Evans, S. Oates, M. Page, S. Zane, P. Schady, A. Breeveld, S. Holland, P. Kuin, M. Still, P. Roming, P. Ward, Jet breaks at the end of the slow decline phase of Swift GRB light curves. Mon. Not. Roy. Astron. Soc. 392, 153-169 (2009). doi:10.1111/j.13652966.2008.13990.x

M. Della Valle, G. Chincarini, N. Panagia, G. Tagliaferri, D. Malesani, V. Testa, D. Fugazza, S. Campana, S. Covino, V. Mangano, L.A. Antonelli, P. D’Avanzo, K. Hurley, I.F. Mirabel, L.J. Pellizza, S. Piranomonte, L. Stella, An enigmatic long-lasting $\gamma$-ray burst not accompanied by a bright supernova. Natur. 444, 1050-1052 (2006). doi:10.1038/nature05374

W. Deng, B. Zhang, Low Energy Spectral Index and $\mathrm{E}_{p}$ Evolution of Quasi-thermal Photosphere Emission of Gamma-Ray Bursts. Astrophys. J. 785, 112 (2014). doi:10.1088/0004- 
$637 \mathrm{X} / 785 / 2 / 112$

C.D. Dermer, A. Atoyan, Collapse of Neutron Stars to Black Holes in Binary Systems: A Model for Short Gamma-Ray Bursts. Astrophys. J. Lett. 643, 13-16 (2006). doi:10.1086/504895

D. Eichler, M. Livio, T. Piran, D.N. Schramm, Nucleosynthesis, neutrino bursts and gammarays from coalescing neutron stars. Natur. 340, 126-128 (1989). doi:10.1038/340126a0

A.D. Falcone, D.N. Burrows, D. Lazzati, S. Campana, S. Kobayashi, B. Zhang, P. Mészáros, K.L. Page, J.A. Kennea, P. Romano, C. Pagani, L. Angelini, A.P. Beardmore, M. Capalbi, G. Chincarini, G. Cusumano, P. Giommi, M.R. Goad, O. Godet, D. Grupe, J.E. Hill, V. La Parola, V. Mangano, A. Moretti, J.A. Nousek, P.T. O'Brien, J.P. Osborne, M. Perri, G. Tagliaferri, A.A. Wells, N. Gehrels, The Giant X-Ray Flare of GRB 050502B: Evidence for Late-Time Internal Engine Activity. Astrophys. J. 641, 1010-1017 (2006). doi:10.1086/500655

Y. Fan, T. Piran, Gamma-ray burst efficiency and possible physical processes shaping the early afterglow. Mon. Not. Roy. Astron. Soc. 369, 197-206 (2006). doi:10.1111/j.13652966.2006.10280.x

Y.Z. Fan, D.M. Wei, Late internal-shock model for bright X-ray flares in gamma-ray burst afterglows and GRB 011121. Mon. Not. Roy. Astron. Soc. 364, 42-46 (2005). doi:10.1111/j.1745-3933.2005.00102.x

Y.-Z. Fan, D. Xu, The X-ray afterglow flat segment in short GRB 051221A: Energy injection from a millisecond magnetar? Mon. Not. Roy. Astron. Soc. 372, 19-22 (2006). doi:10.1111/j.1745-3933.2006.00217.x

Y.Z. Fan, D.M. Wei, C.F. Wang, The very early afterglow powered by ultra-relativistic mildly magnetized outflows. Astron. Astrophys. 424, 477-484 (2004). doi:10.1051/00046361:20041115

Y.-Z. Fan, Z.-G. Dai, Y.-F. Huang, T. Lu, Optical Flash of GRB 990123: Constraints on the Physical Parameters of the Reverse Shock. Chin. J. Astron. Astr. 2, 449-453 (2002)

Y.-Z. Fan, P.H.T. Tam, F.-W. Zhang, Y.-F. Liang, H.-N. He, B. Zhou, R.-Z. Yang, Z.-P. Jin, D.-M. Wei, High-energy Emission of GRB 130427A: Evidence for Inverse Compton Radiation. Astrophys. J. 776, 95 (2013). doi:10.1088/0004-637X/776/2/95

G.J. Fishman, C.A. Meegan, Gamma-Ray Bursts. Annu. Rev. Astron. Astr. 33, 415-458 (1995). doi:10.1146/annurev.aa.33.090195.002215

D.A. Frail, S.R. Kulkarni, L. Nicastro, M. Feroci, G.B. Taylor, The radio afterglow from the $\gamma$-ray burst of 8 May 1997. Natur. 389, 261-263 (1997). doi:10.1038/38451

D.D. Frederiks, V.D. Palshin, R.L. Aptekar, S.V. Golenetskii, T.L. Cline, E.P. Mazets, On the possibility of identifying the short hard burst GRB 051103 with a giant flare from a soft gamma repeater in the M81 group of galaxies. Astronomy Letters 33, 19-24 (2007). doi:10.1134/S1063773707010021

C.L. Fryer, S.E. Woosley, D.H. Hartmann, Formation Rates of Black Hole Accretion Disk Gamma-Ray Bursts. Astrophys. J. 526, 152-177 (1999). doi:10.1086/307992

J.P.U. Fynbo, D. Watson, C.C. Thöne, J. Sollerman, J.S. Bloom, T.M. Davis, J. Hjorth, P. Jakobsson, U.G. Jørgensen, J.F. Graham, A.S. Fruchter, D. Bersier, L. Kewley, A. Cassan, J.M. Castro Cerón, S. Foley, J. Gorosabel, T.C. Hinse, K.D. Horne, B.L. Jensen, S. Klose, D. Kocevski, J.-B. Marquette, D. Perley, E. Ramirez-Ruiz, M.D. Stritzinger, P.M. Vreeswijk, R.A.M. Wijers, K.G. Woller, D. Xu, M. Zub, No supernovae associated with two long-duration $\gamma$-ray bursts. Natur. 444, 1047-1049 (2006). doi:10.1038/nature05375

A. Gal-Yam, D.B. Fox, P.A. Price, E.O. Ofek, M.R. Davis, D.C. Leonard, A.M. Soderberg, B.P. Schmidt, K.M. Lewis, B.A. Peterson, S.R. Kulkarni, E. Berger, S.B. Cenko, R. Sari, K. Sharon, D. Frail, D.-S. Moon, P.J. Brown, A. Cucchiara, F. Harrison, T. Piran, S.E. Persson, P.J. McCarthy, B.E. Penprase, R.A. Chevalier, A.I. MacFadyen, A novel explosive process is required for the $\gamma$-ray burst GRB 060614. Natur. 444, 1053-1055 (2006). doi:10.1038/nature05373

H. Gao, P. Mészáros, Relation between the Intrinsic and Observed Central Engine Activity Time: Implications for Ultra-long GRBs. Astrophys. J. 802, 90 (2015). doi:10.1088/0004$637 \mathrm{X} / 802 / 2 / 90$

H. Gao, B. Zhang, Photosphere Emission from a Hybrid Relativistic Outflow with Arbitrary Dimensionless Entropy and Magnetization in GRBs. Astrophys. J. 801, 103 (2015). doi:10.1088/0004-637X/801/2/103

H. Gao, B. Zhang, H.-J. Lü, Constraints on binary neutron star merger product from short GRB observations. Phys. Rev. D 93(4), 044065 (2016). doi:10.1103/PhysRevD.93.044065

H. Gao, B.-B. Zhang, B. Zhang, Stepwise Filter Correlation Method and Evidence of Su- 
perposed Variability Components in Gamma-Ray Burst Prompt Emission Light Curves. Astrophys. J. 748, 134 (2012). doi:10.1088/0004-637X/748/2/134

H. Gao, W.-H. Lei, Y.-C. Zou, X.-F. Wu, B. Zhang, A complete reference of the analytical synchrotron external shock models of gamma-ray bursts. New Atron. Rev. 57, 141-190 (2013a). doi:10.1016/j.newar.2013.10.001

H. Gao, X. Ding, X.-F. Wu, B. Zhang, Z.-G. Dai, Bright Broadband Afterglows of Gravitational Wave Bursts from Mergers of Binary Neutron Stars. Astrophys. J. 771, 86 (2013b). doi:10.1088/0004-637X/771/2/86

H. Gao, X.-G. Wang, P. Mészáros, B. Zhang, A Morphological Analysis of GammaRay Burst Early-optical Afterglows. Astrophys. J. 810, 160 (2015). doi:10.1088/0004$637 \mathrm{X} / 810 / 2 / 160$

H. Gao, B. Zhang, H.-J. Lü, Y. Li, Searching for magnetar powered merger-novae from short GRBs. ArXiv:1608.03375 (2016)

W.-H. Gao, Y.-Z. Fan, Short-living Supermassive Magnetar Model for the Early X-ray Flares Following Short GRBs. Chin. J. Astron. Astr. 6, 513-516 (2006). doi:10.1088/10099271/6/5/01

N. Gehrels, E. Ramirez-Ruiz, D.B. Fox, Gamma-Ray Bursts in the Swift Era. Annu. Rev. Astron. Astr. 47, 567-617 (2009). doi:10.1146/annurev.astro.46.060407.145147

N. Gehrels, J.P. Norris, S.D. Barthelmy, J. Granot, Y. Kaneko, C. Kouveliotou, C.B. Markwardt, P. Mészáros, E. Nakar, J.A. Nousek, P.T. O’Brien, M. Page, D.M. Palmer, A.M. Parsons, P.W.A. Roming, T. Sakamoto, C.L. Sarazin, P. Schady, M. Stamatikos, S.E. Woosley, A new $\gamma$-ray burst classification scheme from GRB060614. Natur. 444, 10441046 (2006). doi:10.1038/nature05376

B. Gendre, G. Stratta, J.L. Atteia, S. Basa, M. Boër, D.M. Coward, S. Cutini, V. D'Elia, E.J. Howell, A. Klotz, L. Piro, The Ultra-long Gamma-Ray Burst 111209A: The Collapse of a Blue Supergiant? Astrophys. J. 766, 30 (2013). doi:10.1088/0004-637X/766/1/30

F. Genet, F. Daigne, R. Mochkovitch, Can the early X-ray afterglow of gamma-ray bursts be explained by a contribution from the reverse shock? Mon. Not. Roy. Astron. Soc. 381, 732-740 (2007). doi:10.1111/j.1365-2966.2007.12243.x

G. Ghirlanda, G. Ghisellini, L. Nava, D. Burlon, Spectral evolution of Fermi/GBM short gamma-ray bursts. Mon. Not. Roy. Astron. Soc. 410, 47-51 (2011). doi:10.1111/j.17453933.2010.00977.x

G. Ghisellini, G. Ghirlanda, L. Nava, C. Firmani, "Late Prompt" Emission in Gamma-Ray Bursts? Astrophys. J. Lett. 658, 75-78 (2007). doi:10.1086/515570

G. Ghisellini, G. Ghirlanda, L. Nava, A. Celotti, GeV emission from gamma-ray bursts: a radiative fireball? Mon. Not. Roy. Astron. Soc. 403, 926-937 (2010). doi:10.1111/j.13652966.2009.16171.x

D. Giannios, H.C. Spruit, Spectral and timing properties of a dissipative $\gamma$-ray burst photosphere. Astron. Astrophys. 469, 1-9 (2007). doi:10.1051/0004-6361:20066739

S.V. Golenetskii, E.P. Mazets, R.L. Aptekar, V.N. Ilinskii, Correlation between luminosity and temperature in gamma-ray burst sources. Natur. 306, 451-453 (1983). doi:10.1038/306451a0

J. Greiner, P.A. Mazzali, D.A. Kann, T. Krühler, E. Pian, S. Prentice, F. Olivares E., A. Rossi, S. Klose, S. Taubenberger, F. Knust, P.M.J. Afonso, C. Ashall, J. Bolmer, C. Delvaux, R. Diehl, J. Elliott, R. Filgas, J.P.U. Fynbo, J.F. Graham, A.N. Guelbenzu, S. Kobayashi, G. Leloudas, S. Savaglio, P. Schady, S. Schmidl, T. Schweyer, V. Sudilovsky, M. Tanga, A.C. Updike, H. van Eerten, K. Varela, A very luminous magnetar-powered supernova associated with an ultra-long $\gamma$-ray burst. Natur. 523, 189-192 (2015). doi:10.1038/nature14579

D. Gruber, A. Goldstein, V. Weller von Ahlefeld, P. Narayana Bhat, E. Bissaldi, M.S. Briggs, D. Byrne, W.H. Cleveland, V. Connaughton, R. Diehl, G.J. Fishman, G. Fitzpatrick, S. Foley, M. Gibby, M.M. Giles, J. Greiner, S. Guiriec, A.J. van der Horst, A. von Kienlin, C. Kouveliotou, E. Layden, L. Lin, C.A. Meegan, S. McGlynn, W.S. Paciesas, V. Pelassa, R.D. Preece, A. Rau, C.A. Wilson-Hodge, S. Xiong, G. Younes, H.-F. Yu, The Fermi GBM Gamma-Ray Burst Spectral Catalog: Four Years of Data. Astrophys. J. Suppl. 211, 12 (2014). doi:10.1088/0067-0049/211/1/12

D. Grupe, D.N. Burrows, S.K. Patel, C. Kouveliotou, B. Zhang, P. Mészáros, R.A.M. Wijers, N. Gehrels, Jet Breaks in Short Gamma-Ray Bursts. I. The Uncollimated Afterglow of GRB 050724. Astrophys. J. 653, 462-467 (2006). doi:10.1086/508739

D. Grupe, J.A. Nousek, P. Veres, B.-B. Zhang, N. Gehrels, Evidence for New Relations between Gamma-Ray Burst Prompt and X-Ray Afterglow Emission from 9 Years of Swift. 
Astrophys. J. Suppl. 209, 20 (2013). doi:10.1088/0067-0049/209/2/20

C. Guidorzi, R. Margutti, L. Amati, S. Campana, M. Orlandini, P. Romano, M. Stamatikos, G. Tagliaferri, Average power density spectrum of Swift long gamma-ray bursts in the observer and in the source-rest frames. Mon. Not. Roy. Astron. Soc. 422, 1785-1803 (2012). doi:10.1111/j.1365-2966.2012.20758.x

S. Guiriec, V. Connaughton, M.S. Briggs, M. Burgess, F. Ryde, F. Daigne, P. Mészáros, A. Goldstein, J. McEnery, N. Omodei, P.N. Bhat, E. Bissaldi, A. Camero-Arranz, V. Chaplin, R. Diehl, G. Fishman, S. Foley, M. Gibby, M.M. Giles, J. Greiner, D. Gruber, A. von Kienlin, M. Kippen, C. Kouveliotou, S. McBreen, C.A. Meegan, W. Paciesas, R. Preece, A. Rau, D. Tierney, A.J. van der Horst, C. Wilson-Hodge, Detection of a Thermal Spectral Component in the Prompt Emission of GRB 100724B. Astrophys. J. Lett. 727, 33 (2011). doi:10.1088/2041-8205/727/2/L33

S. Guiriec, F. Daigne, R. Hascoët, G. Vianello, F. Ryde, R. Mochkovitch, C. Kouveliotou, S. Xiong, P.N. Bhat, S. Foley, D. Gruber, J.M. Burgess, S. McGlynn, J. McEnery, N. Gehrels, Evidence for a Photospheric Component in the Prompt Emission of the Short GRB 120323A and Its Effects on the GRB Hardness-Luminosity Relation. Astrophys. J. 770, 32 (2013). doi:10.1088/0004-637X/770/1/32

S. Guiriec, R. Mochkovitch, T. Piran, F. Daigne, C. Kouveliotou, J. Racusin, N. Gehrels, J. McEnery, GRB 131014A: A Laboratory for Studying the Thermal-like and Non-thermal Emissions in Gamma-Ray Bursts, and the New $\mathrm{L}^{n T h}{ }_{i}-\mathrm{E}^{n T h, r e s t}{ }_{\text {peak, } i}$ Relation. Astrophys. J. 814, 10 (2015). doi:10.1088/0004-637X/814/1/10

J. Hakkila, T.W. Giblin, R.J. Roiger, D.J. Haglin, W.S. Paciesas, C.A. Meegan, How Sample Completeness Affects Gamma-Ray Burst Classification. Astrophys. J. 582, 320-329 (2003). doi:10.1086/344568

R. Hascoët, F. Daigne, R. Mochkovitch, Accounting for the XRT early steep decay in models of the prompt gamma-ray burst emission. Astron. Astrophys. 542, 29 (2012). doi:10.1051/0004-6361/201219339

S.W. Hawking, Particle creation by black holes. Communications in Mathematical Physics 43, 199-220 (1975). doi:10.1007/BF02345020

H.-N. He, X.-F. Wu, K. Toma, X.-Y. Wang, P. Mészáros, On the High-energy Emission of the Short GRB 090510. Astrophys. J. 733, 22 (2011). doi:10.1088/0004-637X/733/1/22

I. Horváth, A Third Class of Gamma-Ray Bursts? Astrophys. J. 508, 757-759 (1998). doi:10.1086/306416

Y.-D. Hu, E.-W. Liang, S.-Q. Xi, F.-K. Peng, R.-J. Lu, L.-Z. Lü, B. Zhang, Internal Energy Dissipation of Gamma-Ray Bursts Observed with Swift: Precursors, Prompt GammaRays, Extended Emission, and Late X-Ray Flares. Astrophys. J. 789, 145 (2014). doi:10.1088/0004-637X/789/2/145

Y.F. Huang, Z.G. Dai, T. Lu, On the optical light curves of afterglows from jetted gammaray burst ejecta: effects of parameters. Mon. Not. Roy. Astron. Soc. 316, 943-949 (2000). doi:10.1046/j.1365-8711.2000.03683.x

K. Hurley, B.L. Dingus, R. Mukherjee, P. Sreekumar, C. Kouveliotou, C. Meegan, G.J. Fishman, D. Band, L. Ford, D. Bertsch, T. Cline, C. Fichtel, R. Hartman, S. Hunter, D.J. Thompson, G. Kanbach, H. Mayer-Hasselwander, C. von Montigny, M. Sommer, Y. Lin, P. Nolan, P. Michelson, D. Kniffen, J. Mattox, E. Schneid, M. Boer, M. Niel, Detection of a $\gamma$-ray burst of very long duration and very high energy. Natur. 372, 652-654 (1994). doi:10.1038/372652a0

P. Jakobsson, J. Hjorth, J.P.U. Fynbo, D. Watson, K. Pedersen, G. Björnsson, J. Gorosabel, Swift Identification of Dark Gamma-Ray Bursts. Astrophys. J. Lett. 617, 21-24 (2004). doi:10.1086/427089

D.A. Kann, S. Klose, B. Zhang, D. Malesani, E. Nakar, A. Pozanenko, A.C. Wilson, N.R. Butler, P. Jakobsson, S. Schulze, M. Andreev, L.A. Antonelli, I.F. Bikmaev, V. Biryukov, M. Böttcher, R.A. Burenin, J.M. Castro Cerón, A.J. Castro-Tirado, G. Chincarini, B.E. Cobb, S. Covino, P. D'Avanzo, V. D'Elia, M. Della Valle, A. de Ugarte Postigo, Y. Efimov, P. Ferrero, D. Fugazza, J.P.U. Fynbo, M. Gålfalk, F. Grundahl, J. Gorosabel, S. Gupta, S. Guziy, B. Hafizov, J. Hjorth, K. Holhjem, M. Ibrahimov, M. Im, G.L. Israel, M. Jeĺinek, B.L. Jensen, R. Karimov, I.M. Khamitov, Ü. Kiziloğlu, E. Klunko, P. Kubánek, A.S. Kutyrev, P. Laursen, A.J. Levan, F. Mannucci, C.M. Martin, A. Mescheryakov, N. Mirabal, J.P. Norris, J.-E. Ovaldsen, D. Paraficz, E. Pavlenko, S. Piranomonte, A. Rossi, V. Rumyantsev, R. Salinas, A. Sergeev, D. Sharapov, J. Sollerman, B. Stecklum, L. Stella, G. Tagliaferri, N.R. Tanvir, J. Telting, V. Testa, A.C. Updike, A. Volnova, D. Watson, K. 
Wiersema, D. Xu, The Afterglows of Swift-era Gamma-ray Bursts. I. Comparing pre-Swift and Swift-era Long/Soft (Type II) GRB Optical Afterglows. Astrophys. J. 720, 1513-1558 (2010). doi:10.1088/0004-637X/720/2/1513

D.A. Kann, S. Klose, B. Zhang, S. Covino, N.R. Butler, D. Malesani, E. Nakar, A.C. Wilson, L.A. Antonelli, G. Chincarini, B.E. Cobb, P. D'Avanzo, V. D'Elia, M. Della Valle, P. Ferrero, D. Fugazza, J. Gorosabel, G.L. Israel, F. Mannucci, S. Piranomonte, S. Schulze, L. Stella, G. Tagliaferri, K. Wiersema, The Afterglows of Swift-era Gamma-Ray Bursts. II. Type I GRB versus Type II GRB Optical Afterglows. Astrophys. J. 734, 96 (2011). doi:10.1088/0004-637X/734/2/96

K. Kashiyama, D. Nakauchi, Y. Suwa, H. Yajima, T. Nakamura, Luminous Supernovalike UV/Optical/Infrared Transients Associated with Ultra-long Gamma-Ray Bursts from Metal-poor Blue Supergiants. Astrophys. J. 770, 8 (2013). doi:10.1088/0004$637 \mathrm{X} / 770 / 1 / 8$

S. Kobayashi, Light Curves of Gamma-Ray Burst Optical Flashes. Astrophys. J. 545, 807-812 (2000). doi:10.1086/317869

S. Kobayashi, B. Zhang, Early Optical Afterglows from Wind-Type Gamma-Ray Bursts. Astrophys. J. 597, 455-458 (2003a). doi:10.1086/378283

S. Kobayashi, B. Zhang, GRB 021004: Reverse Shock Emission. Astrophys. J. Lett. 582, 75-78 (2003b). doi:10.1086/367691

S. Kobayashi, B. Zhang, The Onset of Gamma-Ray Burst Afterglow. Astrophys. J. 655, 973979 (2007). doi:10.1086/510203

S. Kobayashi, T. Piran, R. Sari, Hydrodynamics of a Relativistic Fireball: The Complete Evolution. Astrophys. J. 513, 669-678 (1999). doi:10.1086/306868

C. Kouveliotou, C.A. Meegan, G.J. Fishman, N.P. Bhat, M.S. Briggs, T.M. Koshut, W.S. Paciesas, G.N. Pendleton, Identification of two classes of gamma-ray bursts. Astrophys. J. Lett. 413, 101-104 (1993). doi:10.1086/186969

S.R. Kulkarni, D.A. Frail, R. Sari, G.H. Moriarty-Schieven, D.S. Shepherd, P. Udomprasert, A.C.S. Readhead, J.S. Bloom, M. Feroci, E. Costa, Discovery of a Radio Flare from GRB 990123. Astrophys. J. Lett. 522, 97-100 (1999). doi:10.1086/312227

P. Kumar, R. Barniol Duran, On the generation of high-energy photons detected by the Fermi Satellite from gamma-ray bursts. Mon. Not. Roy. Astron. Soc. 400, 75-79 (2009). doi:10.1111/j.1745-3933.2009.00766.x

P. Kumar, R. Barniol Duran, External forward shock origin of high-energy emission for three gamma-ray bursts detected by Fermi. Mon. Not. Roy. Astron. Soc. 409, 226-236 (2010). doi:10.1111/j.1365-2966.2010.17274.x

P. Kumar, A. Panaitescu, Steepening of Afterglow Decay for Jets Interacting with Stratified Media. Astrophys. J. Lett. 541, 9-12 (2000). doi:10.1086/312888

P. Kumar, A. Panaitescu, A unified treatment of the gamma-ray burst 021211 and its afterglow. Mon. Not. Roy. Astron. Soc. 346, 905-914 (2003). doi:10.1111/j.1365-2966.2003.07138.x

P. Kumar, B. Zhang, The physics of gamma-ray bursts: relativistic jets. Phys. Rep. 561, 1-109 (2015). doi:10.1016/j.physrep.2014.09.008

P. Kumar, R. Narayan, J.L. Johnson, Mass fall-back and accretion in the central engine of gamma-ray bursts. Mon. Not. Roy. Astron. Soc. 388, 1729-1742 (2008a). doi:10.1111/j.1365-2966.2008.13493.x

P. Kumar, R. Narayan, J.L. Johnson, Properties of Gamma-Ray Burst Progenitor Stars. Science 321, 376 (2008b). doi:10.1126/science.1159003

P.D. Lasky, B. Haskell, V. Ravi, E.J. Howell, D.M. Coward, Nuclear equation of state from observations of short gamma-ray burst remnants. Phys. Rev. D 89(4), 047302 (2014). doi:10.1103/PhysRevD.89.047302

D. Lazzati, Precursor activity in bright, long BATSE gamma-ray bursts. Mon. Not. Roy. Astron. Soc. 357, 722-731 (2005). doi:10.1111/j.1365-2966.2005.08687.x

D. Lazzati, M.C. Begelman, Non-thermal Emission from the Photospheres of Gamma-ray Burst Outflows. I. High-Frequency Tails. Astrophys. J. 725, 1137-1145 (2010). doi:10.1088/0004$637 \mathrm{X} / 725 / 1 / 1137$

D. Lazzati, R. Perna, X-ray flares and the duration of engine activity in gamma-ray bursts. Mon. Not. Roy. Astron. Soc. 375, 46-50 (2007). doi:10.1111/j.1745-3933.2006.00273.x

A.J. Levan, N.R. Tanvir, R.L.C. Starling, K. Wiersema, K.L. Page, D.A. Perley, S. Schulze, G.A. Wynn, R. Chornock, J. Hjorth, S.B. Cenko, A.S. Fruchter, P.T. O'Brien, G.C. Brown, R.L. Tunnicliffe, D. Malesani, P. Jakobsson, D. Watson, E. Berger, D. Bersier, B.E. Cobb, S. Covino, A. Cucchiara, A. de Ugarte Postigo, D.B. Fox, A. Gal-Yam, P. Goldoni, J. 
Gorosabel, L. Kaper, T. Krühler, R. Karjalainen, J.P. Osborne, E. Pian, R. SánchezRamírez, B. Schmidt, I. Skillen, G. Tagliaferri, C. Thöne, O. Vaduvescu, R.A.M.J. Wijers, B.A. Zauderer, A New Population of Ultra-long Duration Gamma-Ray Bursts. Astrophys. J. 781, 13 (2014). doi:10.1088/0004-637X/781/1/13

E.M. Levesque, J.S. Bloom, N.R. Butler, D.A. Perley, S.B. Cenko, J.X. Prochaska, L.J. Kewley, A. Bunker, H.-W. Chen, R. Chornock, A.V. Filippenko, K. Glazebrook, S. Lopez, J. Masiero, M. Modjaz, A. Morgan, D. Poznanski, GRB090426: the environment of a restframe 0.35-s gamma-ray burst at a redshift of 2.609. Mon. Not. Roy. Astron. Soc. 401, 963-972 (2010). doi:10.1111/j.1365-2966.2009.15733.x

A. Li, B. Zhang, N.B. Zhang, H. Gao, B. Qi, T. Liu, Internal X-ray plateau in short GRBs: Signature of supramassive fast-rotating quark stars? ArXiv: 1606.02934 (2016)

L. Li, E.-W. Liang, Q.-W. Tang, J.-M. Chen, S.-Q. Xi, H.-J. Lü, H. Gao, B. Zhang, J. Zhang, S.-X. Yi, R.-J. Lu, L.-Z. Lü, J.-Y. Wei, A Comprehensive Study of Gamma-Ray Burst Optical Emission. I. Flares and Early Shallow-decay Component. Astrophys. J. 758, 27 (2012). doi:10.1088/0004-637X/758/1/27

X. Li, F.-W. Zhang, Q. Yuan, Z.-P. Jin, Y.-Z. Fan, S.-M. Liu, D.-M. Wei, Implications of the Tentative Association between GW150914 and a Fermi-GBM Transient. Astrophys. J. Lett. 827, 16 (2016a). doi:10.3847/2041-8205/827/1/L16

Y. Li, B. Zhang, H.-J. Lü, A Comparative Study of Long and Short GRBs. I. Overlapping Properties. ArXiv:1608.03383 (2016b)

E.-W. Liang, B.-B. Zhang, B. Zhang, A Comprehensive Analysis of Swift XRT Data. II. Diverse Physical Origins of the Shallow Decay Segment. Astrophys. J. 670, 565-583 (2007). doi:10.1086/521870

E.-W. Liang, B.-B. Zhang, M. Stamatikos, B. Zhang, J. Norris, N. Gehrels, J. Zhang, Z.G. Dai, Temporal Profiles and Spectral Lags of XRF 060218. Astrophys. J. Lett. 653, 81-84 (2006a). doi:10.1086/510516

E.W. Liang, B. Zhang, P.T. O'Brien, R. Willingale, L. Angelini, D.N. Burrows, S. Campana, G. Chincarini, A. Falcone, N. Gehrels, M.R. Goad, D. Grupe, S. Kobayashi, P. Mészáros, J.A. Nousek, J.P. Osborne, K.L. Page, G. Tagliaferri, Testing the Curvature Effect and Internal Origin of Gamma-Ray Burst Prompt Emissions and X-Ray Flares with Swift Data. Astrophys. J. 646, 351-357 (2006b). doi:10.1086/504684

E.-W. Liang, J.L. Racusin, B. Zhang, B.-B. Zhang, D.N. Burrows, A Comprehensive Analysis of Swift XRT Data. III. Jet Break Candidates in X-Ray and Optical Afterglow Light Curves. Astrophys. J. 675, 528-552 (2008). doi:10.1086/524701

E.-W. Liang, H.-J. Lü, S.-J. Hou, B.-B. Zhang, B. Zhang, A Comprehensive Analysis of Swift/X-Ray Telescope Data. IV. Single Power-Law Decaying Light Curves Versus Canonical Light Curves and Implications for a Unified Origin of X-Rays. Astrophys. J. 707, 328-342 (2009). doi:10.1088/0004-637X/707/1/328

E.-W. Liang, S.-X. Yi, J. Zhang, H.-J. Lü, B.-B. Zhang, B. Zhang, Constraining Gammaray Burst Initial Lorentz Factor with the Afterglow Onset Feature and Discovery of a Tight $\Gamma_{0}-\mathrm{E}$, iso Correlation. Astrophys. J. 725, 2209-2224 (2010). doi:10.1088/0004$637 \mathrm{X} / 725 / 2 / 2209$

E.-W. Liang, L. Li, H. Gao, B. Zhang, Y.-F. Liang, X.-F. Wu, S.-X. Yi, Z.-G. Dai, Q.-W. Tang, J.-M. Chen, H.-J. Lü, J. Zhang, R.-J. Lu, L.-Z. Lü, J.-Y. Wei, A Comprehensive Study of Gamma-Ray Burst Optical Emission. II. Afterglow Onset and Late Re-brightening Components. Astrophys. J. 774, 13 (2013). doi:10.1088/0004-637X/774/1/13

E. Liang, B. Zhang, F. Virgili, Z.G. Dai, Low-Luminosity Gamma-Ray Bursts as a Unique Population: Luminosity Function, Local Rate, and Beaming Factor. Astrophys. J. 662, 1111-1118 (2007). doi:10.1086/517959

A. Lien, T. Sakamoto, S.D. Barthelmy, W.H. Baumgartner, J.K. Cannizzo, K. Chen, N.R. Collins, J.R. Cummings, N. Gehrels, H.A. Krimm, C.B. Markwardt, D.M. Palmer, M. Stamatikos, E. Troja, T.N. Ukwatta, The Third Swift Burst Alert Telescope Gamma-Ray Burst Catalog. Astrophys. J. 829, 7 (2016). doi:10.3847/0004-637X/829/1/7

R.-Y. Liu, X.-Y. Wang, Modeling the Broadband Emission of GRB 090902B. Astrophys. J. 730, 1 (2011). doi:10.1088/0004-637X/730/1/1

R.-Y. Liu, X.-Y. Wang, X.-F. Wu, Interpretation of the Unprecedentedly Long-lived Highenergy Emission of GRB 130427A. Astrophys. J. Lett. 773, 20 (2013). doi:10.1088/2041$8205 / 773 / 2 /$ L20

A. Loeb, Electromagnetic Counterparts to Black Hole Mergers Detected by LIGO. Astrophys. J. Lett. 819, 21 (2016). doi:10.3847/2041-8205/819/2/L21 
H.-J. Lü, B. Zhang, A Test of the Millisecond Magnetar Central Engine Model of Gamma-Ray Bursts with Swift Data. Astrophys. J. 785, 74 (2014). doi:10.1088/0004-637X/785/1/74

H.-J. Lü, E.-W. Liang, B.-B. Zhang, B. Zhang, A New Classification Method for Gamma-ray Bursts. Astrophys. J. 725, 1965-1970 (2010). doi:10.1088/0004-637X/725/2/1965

H.-J. Lü, B. Zhang, E.-W. Liang, B.-B. Zhang, T. Sakamoto, The 'amplitude' parameter of gamma-ray bursts and its implications for GRB classification. Mon. Not. Roy. Astron. Soc. 442, 1922-1929 (2014). doi:10.1093/mnras/stu982

H.-J. Lü, B. Zhang, W.-H. Lei, Y. Li, P.D. Lasky, The Millisecond Magnetar Central Engine in Short GRBs. Astrophys. J. 805, 89 (2015). doi:10.1088/0004-637X/805/2/89

J. Lü, Y.-C. Zou, W.-H. Lei, B. Zhang, Q. Wu, D.-X. Wang, E.-W. Liang, H.-J. Lü, Lorentzfactor-Isotropic-luminosity/Energy Correlations of Gamma-Ray Bursts and Their Interpretation. Astrophys. J. 751, 49 (2012). doi:10.1088/0004-637X/751/1/49

R.-J. Lu, S.-J. Hou, E.-W. Liang, The $\mathrm{E}$-flux Correlation in the Rising and Decaying Phases of gamma-ray Burst Pulses: Evidence for Viewing Angle Effect? Astrophys. J. 720, 11461154 (2010). doi:10.1088/0004-637X/720/2/1146

R.-J. Lu, J.-J. Wei, E.-W. Liang, B.-B. Zhang, H.-J. Lü, L.-Z. Lü, W.-H. Lei, B. Zhang, A Comprehensive Analysis of Fermi Gamma-Ray Burst Data. II. E ${ }_{p}$ Evolution Patterns and Implications for the Observed Spectrum-Luminosity Relations. Astrophys. J. 756, 112 (2012). doi:10.1088/0004-637X/756/2/112

C. Lundman, A. Pe'er, F. Ryde, A theory of photospheric emission from relativistic, collimated outflows. Mon. Not. Roy. Astron. Soc. 428, 2430-2442 (2013). doi:10.1093/mnras/sts219

N. Lyons, P.T. O'Brien, B. Zhang, R. Willingale, E. Troja, R.L.C. Starling, Can X-ray emission powered by a spinning-down magnetar explain some gamma-ray burst light-curve features? Mon. Not. Roy. Astron. Soc. 402, 705-712 (2010). doi:10.1111/j.1365-2966.2009.15538.x

M. Lyutikov, R. Blandford, Gamma Ray Bursts as Electromagnetic Outflows. Astro$\mathrm{ph} / 0312347$ (2003)

A.I. MacFadyen, S.E. Woosley, Collapsars: Gamma-Ray Bursts and Explosions in "Failed Supernovae". Astrophys. J. 524, 262-289 (1999). doi:10.1086/307790

V. Mangano, V. La Parola, G. Cusumano, T. Mineo, D. Malesani, J. Dyks, S. Campana, M. Capalbi, G. Chincarini, P. Giommi, A. Moretti, M. Perri, P. Romano, G. Tagliaferri, D.N. Burrows, N. Gehrels, O. Godet, S.T. Holland, J.A. Kennea, K.L. Page, J.L. Racusin, P.W.A. Roming, B. Zhang, Swift XRT Observations of the Afterglow of XRF 050416A. Astrophys. J. 654, 403-412 (2007). doi:10.1086/509100

R. Margutti, G. Bernardini, R. Barniol Duran, C. Guidorzi, R.F. Shen, G. Chincarini, On the average gamma-ray burst X-ray flaring activity. Mon. Not. Roy. Astron. Soc. 410, 1064-1075 (2011). doi:10.1111/j.1365-2966.2010.17504.x

A. Maxham, B.-B. Zhang, B. Zhang, Is GeV emission from Gamma-Ray Bursts of external shock origin? Mon. Not. Roy. Astron. Soc. 415, 77-82 (2011). doi:10.1111/j.13652966.2011.18648.x

A. Melandri, S. Kobayashi, C.G. Mundell, C. Guidorzi, A. de Ugarte Postigo, G. Pooley, M. Yoshida, D. Bersier, A.J. Castro-Tirado, M. Jelínek, A. Gomboc, J. Gorosabel, P. Kubánek, M. Bremer, J.M. Winters, I.A. Steele, I. de Gregorio-Monsalvo, R.J. Smith, D. García-Appadoo, A. Sota, A. Lundgren, GRB 090313 and the Origin of Optical Peaks in Gamma-ray Burst Light Curves: Implications for Lorentz Factors and Radio Flares. Astrophys. J. 723, 1331-1342 (2010). doi:10.1088/0004-637X/723/2/1331

P. Mészáros, Theories of Gamma-Ray Bursts. Annu. Rev. Astron. Astr. 40, 137-169 (2002). doi:10.1146/annurev.astro.40.060401.093821

P. Mészáros, Gamma-ray bursts. Reports on Progress in Physics 69, 2259-2321 (2006). doi:10.1088/0034-4885/69/8/R01

P. Meszaros, M.J. Rees, Relativistic fireballs and their impact on external matter - Models for cosmological gamma-ray bursts. Astrophys. J. 405, 278-284 (1993). doi:10.1086/172360

P. Mészáros, M.J. Rees, Optical and Long-Wavelength Afterglow from Gamma-Ray Bursts. Astrophys. J. 476, 232-237 (1997)

P. Mészáros, M.J. Rees, GRB 990123: reverse and internal shock flashes and late afterglow behaviour. Mon. Not. Roy. Astron. Soc. 306, 39-43 (1999). doi:10.1046/j.13658711.1999.02800.x

P. Mészáros, M.J. Rees, Collapsar Jets, Bubbles, and Fe Lines. Astrophys. J. Lett. 556, 37-40 (2001). doi:10.1086/322934

B.D. Metzger, E. Quataert, T.A. Thompson, Short-duration gamma-ray bursts with extended emission from protomagnetar spin-down. Mon. Not. Roy. Astron. Soc. 385, 1455-1460 
(2008). doi:10.1111/j.1365-2966.2008.12923.x

B.D. Metzger, D. Giannios, T.A. Thompson, N. Bucciantini, E. Quataert, The protomagnetar model for gamma-ray bursts. Mon. Not. Roy. Astron. Soc. 413, 2031-2056 (2011). doi:10.1111/j.1365-2966.2011.18280.x

E. Molinari, S.D. Vergani, D. Malesani, S. Covino, P. D'Avanzo, G. Chincarini, F.M. Zerbi, L.A. Antonelli, P. Conconi, V. Testa, G. Tosti, F. Vitali, F. D'Alessio, G. Malaspina, L. Nicastro, E. Palazzi, D. Guetta, S. Campana, P. Goldoni, N. Masetti, E.J.A. Meurs, A. Monfardini, L. Norci, E. Pian, S. Piranomonte, D. Rizzuto, M. Stefanon, L. Stella, G. Tagliaferri, P.A. Ward, G. Ihle, L. Gonzalez, A. Pizarro, P. Sinclaire, J. Valenzuela, REM observations of GRB 060418 and GRB 060607A: the onset of the afterglow and the initial fireball Lorentz factor determination. Astron. Astrophys. 469, 13-16 (2007). doi:10.1051/0004-6361:20077388

B.J. Morsony, D. Lazzati, M.C. Begelman, The Origin and Propagation of Variability in the Outflows of Long-duration Gamma-ray Bursts. Astrophys. J. 723, 267-276 (2010). doi:10.1088/0004-637X/723/1/267

S. Mukherjee, E.D. Feigelson, G. Jogesh Babu, F. Murtagh, C. Fraley, A. Raftery, Three Types of Gamma-Ray Bursts. Astrophys. J. 508, 314-327 (1998). doi:10.1086/306386

K. Murase, K. Asano, T. Terasawa, P. Mészáros, The Role of Stochastic Acceleration in the Prompt Emission of Gamma-Ray Bursts: Application to Hadronic Injection. Astrophys. J. 746, 164 (2012). doi:10.1088/0004-637X/746/2/164

K. Murase, K. Kashiyama, P. Mészáros, I. Shoemaker, N. Senno, Ultrafast Outflows from Black Hole Mergers with a Minidisk. Astrophys. J. Lett. 822, 9 (2016). doi:10.3847/2041$8205 / 822 / 1 /$ L9

E. Nakar, R. Sari, Relativistic Shock Breakouts-A Variety of Gamma-Ray Flares: From Lowluminosity Gamma-Ray Bursts to Type Ia Supernovae. Astrophys. J. 747, 88 (2012). doi:10.1088/0004-637X/747/2/88

R. Narayan, B. Paczynski, T. Piran, Gamma-ray bursts as the death throes of massive binary stars. Astrophys. J. Lett. 395, 83-86 (1992). doi:10.1086/186493

L. Nava, G. Ghirlanda, G. Ghisellini, A. Celotti, Spectral properties of 438 GRBs detected by Fermi/GBM. Astron. Astrophys. 530, 21 (2011). doi:10.1051/0004-6361/201016270

J.P. Norris, J.T. Bonnell, Short Gamma-Ray Bursts with Extended Emission. Astrophys. J. 643, 266-275 (2006). doi:10.1086/502796

J.P. Norris, G.F. Marani, J.T. Bonnell, Connection between Energy-dependent Lags and Peak Luminosity in Gamma-Ray Bursts. Astrophys. J. 534, 248-257 (2000). doi:10.1086/308725

J.P. Norris, G.H. Share, D.C. Messina, B.R. Dennis, U.D. Desai, T.L. Cline, S.M. Matz, E.L. Chupp, Spectral evolution of pulse structures in gamma-ray bursts. Astrophys. J. 301, 213-219 (1986). doi:10.1086/163889

J.A. Nousek, C. Kouveliotou, D. Grupe, K.L. Page, J. Granot, E. Ramirez-Ruiz, S.K. Patel, D.N. Burrows, V. Mangano, S. Barthelmy, A.P. Beardmore, S. Campana, M. Capalbi, G. Chincarini, G. Cusumano, A.D. Falcone, N. Gehrels, P. Giommi, M.R. Goad, O. Godet, C.P. Hurkett, J.A. Kennea, A. Moretti, P.T. O'Brien, J.P. Osborne, P. Romano, G. Tagliaferri, A.A. Wells, Evidence for a Canonical Gamma-Ray Burst Afterglow Light Curve in the Swift XRT Data. Astrophys. J. 642, 389-400 (2006). doi:10.1086/500724

P.T. O'Brien, R. Willingale, J. Osborne, M.R. Goad, K.L. Page, S. Vaughan, E. Rol, A. Beardmore, O. Godet, C.P. Hurkett, A. Wells, B. Zhang, S. Kobayashi, D.N. Burrows, J.A. Nousek, J.A. Kennea, A. Falcone, D. Grupe, N. Gehrels, S. Barthelmy, J. Cannizzo, J. Cummings, J.E. Hill, H. Krimm, G. Chincarini, G. Tagliaferri, S. Campana, A. Moretti, P. Giommi, M. Perri, V. Mangano, V. LaParola, The Early X-Ray Emission from GRBs. Astrophys. J. 647, 1213-1237 (2006). doi:10.1086/505457

W.S. Paciesas, C.A. Meegan, A. von Kienlin, P.N. Bhat, E. Bissaldi, M.S. Briggs, J.M. Burgess, V. Chaplin, V. Connaughton, R. Diehl, G.J. Fishman, G. Fitzpatrick, S. Foley, M. Gibby, M. Giles, A. Goldstein, J. Greiner, D. Gruber, S. Guiriec, A.J. van der Horst, R.M. Kippen, C. Kouveliotou, G. Lichti, L. Lin, S. McBreen, R.D. Preece, A. Rau, D. Tierney, C. WilsonHodge, The Fermi GBM Gamma-Ray Burst Catalog: The First Two Years. Astrophys. J. Suppl. 199, 18 (2012). doi:10.1088/0067-0049/199/1/18

B. Paczynski, Gamma-ray bursters at cosmological distances. Astrophys. J. Lett. 308, 43-46 (1986). doi:10.1086/184740

B. Paczynski, Cosmological gamma-ray bursts. Acta Astronomy 41, 257-267 (1991)

D.M. Palmer, S. Barthelmy, N. Gehrels, R.M. Kippen, T. Cayton, C. Kouveliotou, D. Eichler, R.A.M.J. Wijers, P.M. Woods, J. Granot, Y.E. Lyubarsky, E. Ramirez-Ruiz, L. Barbier, 
M. Chester, J. Cummings, E.E. Fenimore, M.H. Finger, B.M. Gaensler, D. Hullinger, H. Krimm, C.B. Markwardt, J.A. Nousek, A. Parsons, S. Patel, T. Sakamoto, G. Sato, M. Suzuki, J. Tueller, A giant $\gamma$-ray flare from the magnetar SGR 1806 - 20. Natur. 434, 1107-1109 (2005). doi:10.1038/nature03525

A. Panaitescu, P. Kumar, Fundamental Physical Parameters of Collimated Gamma-Ray Burst Afterglows. Astrophys. J. Lett. 560, 49-53 (2001). doi:10.1086/324061

A. Panaitescu, P. Kumar, Properties of Relativistic Jets in Gamma-Ray Burst Afterglows. Astrophys. J. 571, 779-789 (2002). doi:10.1086/340094

A. Panaitescu, P. Mészáros, D. Burrows, J. Nousek, N. Gehrels, P. O’Brien, R. Willingale, Evidence for chromatic X-ray light-curve breaks in Swift gamma-ray burst afterglows and their theoretical implications. Mon. Not. Roy. Astron. Soc. 369, 2059-2064 (2006). doi:10.1111/j.1365-2966.2006.10453.x

A. Pe'er, P. Mészáros, M.J. Rees, The Observable Effects of a Photospheric Component on GRB and XRF Prompt Emission Spectrum. Astrophys. J. 642, 995-1003 (2006). doi:10.1086/501424

A. Pe'Er, B.-B. Zhang, F. Ryde, S. McGlynn, B. Zhang, R.D. Preece, C. Kouveliotou, The connection between thermal and non-thermal emission in gamma-ray bursts: general considerations and GRB 090902B as a case study. Mon. Not. Roy. Astron. Soc. 420, 468-482 (2012). doi:10.1111/j.1365-2966.2011.20052.x

R. Perna, D. Lazzati, B. Giacomazzo, Short Gamma-Ray Bursts from the Merger of Two Black Holes. Astrophys. J. Lett. 821, 18 (2016). doi:10.3847/2041-8205/821/1/L18

T. Piran, Gamma-ray bursts and the fireball model. Phys. Rep. 314, 575-667 (1999). doi:10.1016/S0370-1573(98)00127-6

T. Piran, The physics of gamma-ray bursts. Reviews of Modern Physics 76, 1143-1210 (2004). doi:10.1103/RevModPhys.76.1143

R. Popham, S.E. Woosley, C. Fryer, Hyperaccreting Black Holes and Gamma-Ray Bursts. Astrophys. J. 518, 356-374 (1999). doi:10.1086/307259

R.D. Preece, M.S. Briggs, R.S. Mallozzi, G.N. Pendleton, W.S. Paciesas, D.L. Band, The BATSE Gamma-Ray Burst Spectral Catalog. I. High Time Resolution Spectroscopy of Bright Bursts Using High Energy Resolution Data. Astrophys. J. Suppl. 126, 19-36 (2000). doi:10.1086/313289

B. Qin, X.-P. Wu, M.-C. Chu, L.-Z. Fang, J.-Y. Hu, The Collapse of Neutron Stars in HighMass Binaries as the Energy Source for the Gamma-Ray Bursts. Astrophys. J. Lett. 494, 57-59 (1998). doi:10.1086/311152

Y. Qin, E.-W. Liang, Y.-F. Liang, S.-X. Yi, L. Lin, B.-B. Zhang, J. Zhang, H.-J. Lü, R.-J. Lu, L.-Z. Lü, B. Zhang, A Comprehensive Analysis of Fermi Gamma-Ray Burst Data. III. Energy-dependent T 90 Distributions of GBM GRBs and Instrumental Selection Effect on Duration Classification. Astrophys. J. 763, 15 (2013). doi:10.1088/0004-637X/763/1/15

J.L. Racusin, S.V. Karpov, M. Sokolowski, J. Granot, X.F. Wu, V. Pal'Shin, S. Covino, A.J. van der Horst, S.R. Oates, P. Schady, R.J. Smith, J. Cummings, R.L.C. Starling, L.W. Piotrowski, B. Zhang, P.A. Evans, S.T. Holland, K. Malek, M.T. Page, L. Vetere, R. Margutti, C. Guidorzi, A.P. Kamble, P.A. Curran, A. Beardmore, C. Kouveliotou, L. Mankiewicz, A. Melandri, P.T. O'Brien, K.L. Page, T. Piran, N.R. Tanvir, G. Wrochna, R.L. Aptekar, S. Barthelmy, C. Bartolini, G.M. Beskin, S. Bondar, M. Bremer, S. Campana, A. Castro-Tirado, A. Cucchiara, M. Cwiok, P. D’Avanzo, V. D’Elia, M. Della Valle, A. de Ugarte Postigo, W. Dominik, A. Falcone, F. Fiore, D.B. Fox, D.D. Frederiks, A.S. Fruchter, D. Fugazza, M.A. Garrett, N. Gehrels, S. Golenetskii, A. Gomboc, J. Gorosabel, G. Greco, A. Guarnieri, S. Immler, M. Jelinek, G. Kasprowicz, V. La Parola, A.J. Levan, V. Mangano, E.P. Mazets, E. Molinari, A. Moretti, K. Nawrocki, P.P. Oleynik, J.P. Osborne, C. Pagani, S.B. Pandey, Z. Paragi, M. Perri, A. Piccioni, E. Ramirez-Ruiz, P.W.A. Roming, I.A. Steele, R.G. Strom, V. Testa, G. Tosti, M.V. Ulanov, K. Wiersema, R.A.M.J. Wijers, J.M. Winters, A.F. Zarnecki, F. Zerbi, P. Mészáros, G. Chincarini, D.N. Burrows, Broadband observations of the naked-eye $\gamma$-ray burst GRB080319B. Natur. 455, 183-188 (2008). doi:10.1038/nature07270

J.L. Racusin, E.W. Liang, D.N. Burrows, A. Falcone, T. Sakamoto, B.B. Zhang, B. Zhang, P. Evans, J. Osborne, Jet Breaks and Energetics of Swift Gamma-Ray Burst X-Ray Afterglows. Astrophys. J. 698, 43-74 (2009). doi:10.1088/0004-637X/698/1/43

M.J. Rees, P. Meszaros, Relativistic fireballs - Energy conversion and time-scales. Mon. Not. Roy. Astron. Soc. 258, 41-43 (1992). doi:10.1093/mnras/258.1.41P

M.J. Rees, P. Meszaros, Unsteady outflow models for cosmological gamma-ray bursts. Astro- 
phys. J. Lett. 430, 93-96 (1994). doi:10.1086/187446

M.J. Rees, P. Mészáros, Refreshed Shocks and Afterglow Longevity in Gamma-Ray Bursts. Astrophys. J. Lett. 496, 1-4 (1998). doi:10.1086/311244

M.J. Rees, P. Mészáros, Dissipative Photosphere Models of Gamma-Ray Bursts and X-Ray Flashes. Astrophys. J. 628, 847-852 (2005). doi:10.1086/430818

L. Resmi, B. Zhang, Gamma-ray Burst Reverse Shock Emission in Early Radio Afterglows. Astrophys. J. 825, 48 (2016). doi:10.3847/0004-637X/825/1/48

L. Rezzolla, B. Giacomazzo, L. Baiotti, J. Granot, C. Kouveliotou, M.A. Aloy, The Missing Link: Merging Neutron Stars Naturally Produce Jet-like Structures and Can Power Short Gamma-ray Bursts. Astrophys. J. Lett. 732, 6 (2011). doi:10.1088/2041-8205/732/1/L6

J.E. Rhoads, The Dynamics and Light Curves of Beamed Gamma-Ray Burst Afterglows. Astrophys. J. 525, 737-749 (1999). doi:10.1086/307907

E. Rol, R.A.M.J. Wijers, C. Kouveliotou, L. Kaper, Y. Kaneko, How Special Are Dark GammaRay Bursts: A Diagnostic Tool. Astrophys. J. 624, 868-879 (2005). doi:10.1086/429082

P. Romano, A. Moretti, P.L. Banat, D.N. Burrows, S. Campana, G. Chincarini, S. Covino, D. Malesani, G. Tagliaferri, S. Kobayashi, B. Zhang, A.D. Falcone, L. Angelini, S. Barthelmy, A.P. Beardmore, M. Capalbi, G. Cusumano, P. Giommi, M.R. Goad, O. Godet, D. Grupe, J.E. Hill, J.A. Kennea, V. La Parola, V. Mangano, P. Mészáros, D.C. Morris, J.A. Nousek, P.T. O'Brien, J.P. Osborne, A. Parsons, M. Perri, C. Pagani, K.L. Page, A.A. Wells, N. Gehrels, X-ray flare in XRF 050406: evidence for prolonged engine activity. Astron. Astrophys. 450, 59-68 (2006). doi:10.1051/0004-6361:20054172

S. Rosswog, E. Ramirez-Ruiz, M.B. Davies, High-resolution calculations of merging neutron stars - III. Gamma-ray bursts. Mon. Not. Roy. Astron. Soc. 345, 1077-1090 (2003). doi:10.1046/j.1365-2966.2003.07032.x

A. Rowlinson, P.T. O'Brien, N.R. Tanvir, B. Zhang, P.A. Evans, N. Lyons, A.J. Levan, R. Willingale, K.L. Page, O. Onal, D.N. Burrows, A.P. Beardmore, T.N. Ukwatta, E. Berger, J. Hjorth, A.S. Fruchter, R.L. Tunnicliffe, D.B. Fox, A. Cucchiara, The unusual X-ray emission of the short Swift GRB 090515: evidence for the formation of a magnetar? Mon. Not. Roy. Astron. Soc. 409, 531-540 (2010). doi:10.1111/j.1365-2966.2010.17354.x

A. Rowlinson, P.T. O'Brien, B.D. Metzger, N.R. Tanvir, A.J. Levan, Signatures of magnetar central engines in short GRB light curves. Mon. Not. Roy. Astron. Soc. 430, 1061-1087 (2013). doi:10.1093/mnras/sts683

R. Ruffini, J.A. Rueda, M. Muccino, Y. Aimuratov, L.M. Becerra, C.L. Bianco, M. Kovacevic, R. Moradi, F.G. Oliveira, G.B. Pisani, Y. Wang, On the classification of GRBs and their occurrence rates. ArXiv:1602.02732 (2016)

F. Ryde, Is Thermal Emission in Gamma-Ray Bursts Ubiquitous? Astrophys. J. Lett. 625, 95-98 (2005). doi:10.1086/431239

F. Ryde, M. Axelsson, B.B. Zhang, S. McGlynn, A. Pe'er, C. Lundman, S. Larsson, M. Battelino, B. Zhang, E. Bissaldi, J. Bregeon, M.S. Briggs, J. Chiang, F. de Palma, S. Guiriec, J. Larsson, F. Longo, S. McBreen, N. Omodei, V. Petrosian, R. Preece, A.J. van der Horst, Identification and Properties of the Photospheric Emission in GRB090902B. Astrophys. J. Lett. 709, 172-177 (2010). doi:10.1088/2041-8205/709/2/L172

T. Sakamoto, D.Q. Lamb, N. Kawai, A. Yoshida, C. Graziani, E.E. Fenimore, T.Q. Donaghy, M. Matsuoka, M. Suzuki, G. Ricker, J.-L. Atteia, Y. Shirasaki, T. Tamagawa, K. Torii, M. Galassi, J. Doty, R. Vanderspek, G.B. Crew, J. Villasenor, N. Butler, G. Prigozhin, J.G. Jernigan, C. Barraud, M. Boer, J.-P. Dezalay, J.-F. Olive, K. Hurley, A. Levine, G. Monnelly, F. Martel, E. Morgan, S.E. Woosley, T. Cline, J. Braga, R. Manchanda, G. Pizzichini, K. Takagishi, M. Yamauchi, Global Characteristics of X-Ray Flashes and XRay-Rich Gamma-Ray Bursts Observed by HETE-2. Astrophys. J. 629, 311-327 (2005). doi:10.1086/431235

T. Sakamoto, S.D. Barthelmy, W.H. Baumgartner, J.R. Cummings, E.E. Fenimore, N. Gehrels, H.A. Krimm, C.B. Markwardt, D.M. Palmer, A.M. Parsons, G. Sato, M. Stamatikos, J. Tueller, T.N. Ukwatta, B. Zhang, The Second Swift Burst Alert Telescope Gamma-Ray Burst Catalog. Astrophys. J. Suppl. 195, 2 (2011). doi:10.1088/0067-0049/195/1/2

R. Sari, P. Mészáros, Impulsive and Varying Injection in Gamma-Ray Burst Afterglows. Astrophys. J. Lett. 535, 33-37 (2000). doi:10.1086/312689

R. Sari, T. Piran, Hydrodynamic Timescales and Temporal Structure of Gamma-Ray Bursts. Astrophys. J. Lett. 455, 143 (1995). doi:10.1086/309835

R. Sari, T. Piran, GRB 990123: The Optical Flash and the Fireball Model. Astrophys. J. Lett. 517, 109-112 (1999a). doi:10.1086/312039 
R. Sari, T. Piran, Predictions for the Very Early Afterglow and the Optical Flash. Astrophys. J. 520, 641-649 (1999b). doi:10.1086/307508

R. Sari, T. Piran, J.P. Halpern, Jets in Gamma-Ray Bursts. Astrophys. J. Lett. 519, 17-20 (1999). doi:10.1086/312109

R. Sari, T. Piran, R. Narayan, Spectra and Light Curves of Gamma-Ray Burst Afterglows. Astrophys. J. Lett. 497, 17-20 (1998). doi:10.1086/311269

A.M. Soderberg, S.R. Kulkarni, E. Nakar, E. Berger, P.B. Cameron, D.B. Fox, D. Frail, A. GalYam, R. Sari, S.B. Cenko, M. Kasliwal, R.A. Chevalier, T. Piran, P.A. Price, B.P. Schmidt, G. Pooley, D.-S. Moon, B.E. Penprase, E. Ofek, A. Rau, N. Gehrels, J.A. Nousek, D.N. Burrows, S.E. Persson, P.J. McCarthy, Relativistic ejecta from X-ray flash XRF 060218 and the rate of cosmic explosions. Natur. 442, 1014-1017 (2006). doi:10.1038/nature05087

H. Sun, B. Zhang, Z. Li, Extragalactic High-energy Transients: Event Rate Densities and Luminosity Functions. Astrophys. J. 812, 33 (2015). doi:10.1088/0004-637X/812/1/33

P.-H.T. Tam, Q.-W. Tang, S.-J. Hou, R.-Y. Liu, X.-Y. Wang, Discovery of an Extra Hard Spectral Component in the High-energy Afterglow Emission of GRB 130427A. Astrophys. J. Lett. 771, 13 (2013). doi:10.1088/2041-8205/771/1/L13

N.R. Tanvir, R. Chapman, A.J. Levan, R.S. Priddey, An origin in the local Universe for some short $\gamma$-ray bursts. Natur. 438, 991-993 (2005). doi:10.1038/nature04310

C.C. Thöne, A. de Ugarte Postigo, C.L. Fryer, K.L. Page, J. Gorosabel, M.A. Aloy, D.A. Perley, C. Kouveliotou, H.T. Janka, P. Mimica, J.L. Racusin, H. Krimm, J. Cummings, S.R. Oates, S.T. Holland, M.H. Siegel, M. de Pasquale, E. Sonbas, M. Im, W.-K. Park, D.A. Kann, S. Guziy, L.H. García, A. Llorente, K. Bundy, C. Choi, H. Jeong, H. Korhonen, P. Kubànek, J. Lim, A. Moskvitin, T. Muñoz-Darias, S. Pak, I. Parrish, The unusual $\gamma$-ray burst GRB 101225A from a helium star/neutron star merger at redshift 0.33. Natur. 480, 72-74 (2011). doi:10.1038/nature10611

K. Toma, X.-F. Wu, P. Mészáros, Photosphere-internal shock model of gamma-ray bursts: case studies of Fermi/LAT bursts. Mon. Not. Roy. Astron. Soc. 415, 1663-1680 (2011). doi:10.1111/j.1365-2966.2011.18807.x

E. Troja, G. Cusumano, P.T. O'Brien, B. Zhang, B. Sbarufatti, V. Mangano, R. Willingale, G. Chincarini, J.P. Osborne, F.E. Marshall, D.N. Burrows, S. Campana, N. Gehrels, C. Guidorzi, H.A. Krimm, V. La Parola, E.W. Liang, T. Mineo, A. Moretti, K.L. Page, P. Romano, G. Tagliaferri, B.B. Zhang, M.J. Page, P. Schady, Swift Observations of GRB 070110: An Extraordinary X-Ray Afterglow Powered by the Central Engine. Astrophys. J. 665, 599-607 (2007). doi:10.1086/519450

Z.L. Uhm, A.M. Beloborodov, On the Mechanism of Gamma-Ray Burst Afterglows. Astrophys. J. Lett. 665, 93-96 (2007). doi:10.1086/519837

Z.L. Uhm, B. Zhang, Dynamics and Afterglow Light Curves of Gamma-Ray Burst Blast Waves Encountering a Density Bump or Void. Astrophys. J. 789, 39 (2014a). doi:10.1088/0004$637 \mathrm{X} / 789 / 1 / 39$

Z.L. Uhm, B. Zhang, Fast-cooling synchrotron radiation in a decaying magnetic field and $\gamma$-ray burst emission mechanism. Nature Physics 10, 351-356 (2014b). doi:10.1038/nphys2932

Z.L. Uhm, B. Zhang, Toward an Understanding of GRB Prompt Emission Mechanism. I. The Origin of Spectral Lags. Astrophys. J. 825, 97 (2016). doi:10.3847/0004-637X/825/2/97

Z.L. Uhm, B. Zhang, R. Hascoët, F. Daigne, R. Mochkovitch, I.H. Park, Dynamics and Afterglow Light Curves of Gamma-Ray Burst Blast Waves with a Long-lived Reverse Shock. Astrophys. J. 761, 147 (2012). doi:10.1088/0004-637X/761/2/147

T.N. Ukwatta, D.R. Stump, J.T. Linnemann, J.H. MacGibbon, S.S. Marinelli, T. Yapici, K. Tollefson, Primordial Black Holes: Observational characteristics of the final evaporation. Astroparticle Physics 80, 90-114 (2016). doi:10.1016/j.astropartphys.2016.03.007

V.V. Usov, Millisecond pulsars with extremely strong magnetic fields as a cosmological source of gamma-ray bursts. Natur. 357, 472-474 (1992). doi:10.1038/357472a0

J. van Paradijs, C. Kouveliotou, R.A.M.J. Wijers, Gamma-Ray Burst Afterglows. Annu. Rev. Astron. Astr. 38, 379-425 (2000). doi:10.1146/annurev.astro.38.1.379

J. van Paradijs, P.J. Groot, T. Galama, C. Kouveliotou, R.G. Strom, J. Telting, R.G.M. Rutten, G.J. Fishman, C.A. Meegan, M. Pettini, N. Tanvir, J. Bloom, H. Pedersen, H.U. Nørdgaard-Nielsen, M. Linden-Vørnle, J. Melnick, G. van der Steene, M. Bremer, R. Naber, J. Heise, J. in't Zand, E. Costa, M. Feroci, L. Piro, F. Frontera, G. Zavattini, L. Nicastro, E. Palazzi, K. Bennett, L. Hanlon, A. Parmar, Transient optical emission from the error box of the $\gamma$-ray burst of 28 February 1997. Natur. 386, 686-689 (1997). doi:10.1038/386686a0

P. Veres, R.D. Preece, A. Goldstein, P. Mészáros, E. Burns, V. Connaughton, Gravitational- 
wave Observations May Constrain Gamma-Ray Burst Models: The Case of GW150914GBM. Astrophys. J. Lett. 827, 34 (2016). doi:10.3847/2041-8205/827/2/L34

W.T. Vestrand, J.A. Wren, P.R. Wozniak, R. Aptekar, S. Golentskii, V. Pal'Shin, T. Sakamoto, R.R. White, S. Evans, D. Casperson, E. Fenimore, Energy input and response from prompt and early optical afterglow emission in $\gamma$-ray bursts. Natur. 442, 172-175 (2006). doi:10.1038/nature04913

F.J. Virgili, E.-W. Liang, B. Zhang, Low-luminosity gamma-ray bursts as a distinct GRB population: a firmer case from multiple criteria constraints. Mon. Not. Roy. Astron. Soc. 392, 91-103 (2009). doi:10.1111/j.1365-2966.2008.14063.x

F.J. Virgili, C.G. Mundell, V. Pal'shin, C. Guidorzi, R. Margutti, A. Melandri, R. Harrison, S. Kobayashi, R. Chornock, A. Henden, A.C. Updike, S.B. Cenko, N.R. Tanvir, I.A. Steele, A. Cucchiara, A. Gomboc, A. Levan, Z. Cano, C.J. Mottram, N.R. Clay, D. Bersier, D. Kopač, J. Japelj, A.V. Filippenko, W. Li, D. Svinkin, S. Golenetskii, D.H. Hartmann, P.A. Milne, G. Williams, P.T. O'Brien, D.B. Fox, E. Berger, GRB 091024A and the Nature of Ultralong Gamma-Ray Bursts. Astrophys. J. 778, 54 (2013). doi:10.1088/0004-637X/778/1/54

I. Vurm, A.M. Beloborodov, J. Poutanen, Gamma-Ray Bursts from Magnetized Collisionally Heated Jets. Astrophys. J. 738, 77 (2011). doi:10.1088/0004-637X/738/1/77

X.-G. Wang, B. Zhang, E.-W. Liang, H. Gao, L. Li, C.-M. Deng, S.-M. Qin, Q.-W. Tang, D.A. Kann, F. Ryde, P. Kumar, How Bad or Good Are the External Forward Shock Afterglow Models of Gamma-Ray Bursts? Astrophys. J. Suppl. 219, 9 (2015). doi:10.1088/00670049/219/1/9

R. Willingale, P.T. O'Brien, J.P. Osborne, O. Godet, K.L. Page, M.R. Goad, D.N. Burrows, B. Zhang, E. Rol, N. Gehrels, G. Chincarini, Testing the Standard Fireball Model of Gamma-Ray Bursts Using Late X-Ray Afterglows Measured by Swift. Astrophys. J. 662, 1093-1110 (2007). doi:10.1086/517989

S.E. Woosley, Gamma-ray bursts from stellar mass accretion disks around black holes. Astrophys. J. 405, 273-277 (1993). doi:10.1086/172359

S.E. Woosley, J.S. Bloom, The Supernova Gamma-Ray Burst Connection. Annu. Rev. Astron. Astr. 44, 507-556 (2006). doi:10.1146/annurev.astro.43.072103.150558

X.F. Wu, Z.G. Dai, Y.F. Huang, T. Lu, Optical flashes and very early afterglows in wind environments. Mon. Not. Roy. Astron. Soc. 342, 1131-1138 (2003). doi:10.1046/j.13658711.2003.06602.x

L.-P. Xin, E.-W. Liang, J.-Y. Wei, B. Zhang, H.-J. Lv, W.-K. Zheng, Y. Urata, M. Im, J. Wang, Y.-L. Qiu, J.-S. Deng, K.-Y. Huang, J.-Y. Hu, Y. Jeon, H.-L. Li, X.-H. Han, Probing the nature of high-z short GRB 090426 with its early optical and X-ray afterglows. Mon. Not. Roy. Astron. Soc. 410, 27-32 (2011). doi:10.1111/j.1365-2966.2010.17419.x

R.-R. Xue, Y.-Z. Fan, D.-M. Wei, The initial Lorentz factors of fireballs inferred from the early X-ray data of SWIFT GRBs. Astron. Astrophys. 498, 671-676 (2009). doi:10.1051/0004$6361 / 200811361$

R. Yamazaki, K. Asano, Y. Ohira, Electromagnetic afterglows associated with gamma-ray emission coincident with binary black hole merger event GW150914. Progress of Theoretical and Experimental Physics 2016(5), 051-01 (2016). doi:10.1093/ptep/ptw042

S.A. Yost, F.A. Harrison, R. Sari, D.A. Frail, A Study of the Afterglows of Four GammaRay Bursts: Constraining the Explosion and Fireball Model. Astrophys. J. 597, 459-473 (2003). doi:10.1086/378288

Y.W. Yu, Z.G. Dai, Shallow decay phase of GRB X-ray afterglows from relativistic wind bubbles. Astron. Astrophys. 470, 119-122 (2007). doi:10.1051/0004-6361:20077053

Y.-W. Yu, B. Zhang, H. Gao, Bright "Merger-nova" from the Remnant of a Neutron Star Binary Merger: A Signature of a Newly Born, Massive, Millisecond Magnetar. Astrophys. J. Lett. 776, 40 (2013). doi:10.1088/2041-8205/776/2/L40

B. Zhang, Astrophysics: A burst of new ideas. Natur. 444, 1010-1011 (2006). doi:10.1038/4441010a

B. Zhang, Gamma-Ray Bursts in the Swift Era. Chin. J. Astron. Astr. 7, 1-50 (2007). doi:10.1088/1009-9271/7/1/01

B. Zhang, Open questions in GRB physics. Comptes Rendus Physique 12, 206-225 (2011). doi:10.1016/j.crhy.2011.03.004

B. Zhang, Early X-Ray and Optical Afterglow of Gravitational Wave Bursts from Mergers of Binary Neutron Stars. Astrophys. J. Lett. 763, 22 (2013). doi:10.1088/2041$8205 / 763 / 1 / \mathrm{L} 22$

B. Zhang, Mergers of Charged Black Holes: Gravitational-wave Events, Short Gamma-Ray 
Bursts, and Fast Radio Bursts. Astrophys. J. Lett. 827, 31 (2016). doi:10.3847/2041$8205 / 827 / 2 /$ L31

B. Zhang, S. Kobayashi, Gamma-Ray Burst Early Afterglows: Reverse Shock Emission from an Arbitrarily Magnetized Ejecta. Astrophys. J. 628, 315-334 (2005). doi:10.1086/429787

B. Zhang, P. Kumar, Model-Dependent High-Energy Neutrino Flux from Gamma-Ray Bursts. Physical Review Letters 110(12), 121101 (2013). doi:10.1103/PhysRevLett.110.121101

B. Zhang, P. Mészáros, Gamma-Ray Burst Afterglow with Continuous Energy Injection: Signature of a Highly Magnetized Millisecond Pulsar. Astrophys. J. Lett. 552, 35-38 (2001). doi: $10.1086 / 320255$

B. Zhang, P. Mészáros, Gamma-Ray Bursts with Continuous Energy Injection and Their Afterglow Signature. Astrophys. J. 566, 712-722 (2002). doi:10.1086/338247

B. Zhang, P. Mészáros, Gamma-Ray Bursts: progress, problems, prospects. International Journal of Modern Physics A 19, 2385-2472 (2004). doi:10.1142/S0217751X0401746X

B. Zhang, H. Yan, The Internal-collision-induced Magnetic Reconnection and Turbulence (ICMART) Model of Gamma-ray Bursts. Astrophys. J. 726, 90 (2011). doi:10.1088/0004$637 \mathrm{X} / 726 / 2 / 90$

B. Zhang, B. Zhang, Gamma-Ray Burst Prompt Emission Light Curves and Power Density Spectra in the ICMART Model. Astrophys. J. 782, 92 (2014). doi:10.1088/0004$637 \mathrm{X} / 782 / 2 / 92$

B. Zhang, S. Kobayashi, P. Mészáros, Gamma-Ray Burst Early Optical Afterglows: Implications for the Initial Lorentz Factor and the Central Engine. Astrophys. J. 595, 950-954 (2003). doi: $10.1086 / 377363$

B.-B. Zhang, E.-W. Liang, B. Zhang, A Comprehensive Analysis of Swift XRT Data. I. Apparent Spectral Evolution of Gamma-Ray Burst X-Ray Tails. Astrophys. J. 666, 1002-1011 (2007). doi:10.1086/519548

B.-B. Zhang, B. Zhang, E.-W. Liang, X.-Y. Wang, Curvature Effect of a Non-Power-Law Spectrum and Spectral Evolution of GRB X-Ray Tails. Astrophys. J. Lett. 690, 10-13 (2009). doi:10.1088/0004-637X/690/1/L10

B.-B. Zhang, B. Zhang, E.-W. Liang, Y.-Z. Fan, X.-F. Wu, A. Pe'er, A. Maxham, H. Gao, Y.-M. Dong, A Comprehensive Analysis of Fermi Gamma-ray Burst Data. I. Spectral Components and the Possible Physical Origins of LAT/GBM GRBs. Astrophys. J. 730, 141 (2011). doi:10.1088/0004-637X/730/2/141

B.-B. Zhang, B. Zhang, K. Murase, V. Connaughton, M.S. Briggs, How Long does a Burst Burst? Astrophys. J. 787, 66 (2014). doi:10.1088/0004-637X/787/1/66

B.-B. Zhang, Z.L. Uhm, V. Connaughton, M.S. Briggs, B. Zhang, Synchrotron Origin of the Typical GRB Band Function: A Case Study of GRB 130606B. Astrophys. J. 816, 72 (2016). doi:10.3847/0004-637X/816/2/72

B. Zhang, Y.Z. Fan, J. Dyks, S. Kobayashi, P. Mészáros, D.N. Burrows, J.A. Nousek, N. Gehrels, Physical Processes Shaping Gamma-Ray Burst X-Ray Afterglow Light Curves: Theoretical Implications from the Swift X-Ray Telescope Observations. Astrophys. J. 642, 354-370 (2006). doi:10.1086/500723

B. Zhang, E. Liang, K.L. Page, D. Grupe, B.-B. Zhang, S.D. Barthelmy, D.N. Burrows, S. Campana, G. Chincarini, N. Gehrels, S. Kobayashi, P. Mészáros, A. Moretti, J.A. Nousek, P.T. O'Brien, J.P. Osborne, P.W.A. Roming, T. Sakamoto, P. Schady, R. Willingale, GRB Radiative Efficiencies Derived from the Swift Data: GRBs versus XRFs, Long versus Short. Astrophys. J. 655, 989-1001 (2007a). doi:10.1086/510110

B. Zhang, B.-B. Zhang, E.-W. Liang, N. Gehrels, D.N. Burrows, P. Mészáros, Making a Short Gamma-Ray Burst from a Long One: Implications for the Nature of GRB 060614. Astrophys. J. Lett. 655, 25-28 (2007b). doi:10.1086/511781

B. Zhang, B.-B. Zhang, F.J. Virgili, E.-W. Liang, D.A. Kann, X.-F. Wu, D. Proga, H.-J. Lv, K. Toma, P. Mészáros, D.N. Burrows, P.W.A. Roming, N. Gehrels, Discerning the Physical Origins of Cosmological Gamma-ray Bursts Based on Multiple Observational Criteria: The Cases of $\mathrm{z}=6.7$ GRB 080913, $\mathrm{z}=8.2 \mathrm{GRB}$ 090423, and Some Short/Hard GRBs. Astrophys. J. 703, 1696-1724 (2009). doi:10.1088/0004-637X/703/2/1696

F.-W. Zhang, L. Shao, J.-Z. Yan, D.-M. Wei, Revisiting the Long/Soft-Short/Hard Classification of Gamma-Ray Bursts in the Fermi Era. Astrophys. J. 750, 88 (2012). doi:10.1088/0004-637X/750/2/88 